\title{
HOCHSCHILD COHOMOLOGY OF ALGEBRAS OF SEMIDIHEDRAL TYPE. I. GROUP ALGEBRAS OF SEMIDIHEDRAL GROUPS
}

\author{
A. I. GENERALOV
}

\begin{abstract}
For a family of local algebras of semidihedral type over an algebraically closed field of characteristic 2, the Hochschild cohomology algebra is described in terms of generators and relations. The calculations are based on the construction of a bimodule resolution for the algebras in question. As a consequence, the Hochschild cohomology algebra is described for the group algebras of semidihedral groups.
\end{abstract}

\section{INTRODUCTION}

In recent years, interest has grown considerably in the investigation of the Hochschild cohomology algebra, and appreciable success has been made for the case of finite-dimensional algebras over fields. In [1], a description was obtained of the Hochschild cohomology algebra for the symmetric group $S_{3}$ over the field $\mathbb{F}_{3}$, and also for the alternating group $A_{4}$ and the dihedral 2-groups over the field $\mathbb{F}_{2}$. In [2], the Hochschild cohomology algebra $\mathrm{HH}^{*}(R)$ was described in the case where $R$ is a self-injective Nakayama algebra. In [3, a description of the Hochschild cohomology algebra was given for algebras of dihedral type in the family $D(3 \mathcal{K})$ over an algebraically closed ground field of characteristic two. In that paper, unlike [1, we applied a direct method of constructing a bimodule resolution for the corresponding algebras, with subsequent use of this resolution in the calculation of Hochschild cohomology groups and multiplication in the cohomology algebra. We recall that algebras of dihedral, semidihedral, and quaternion types appeared in the work of K. Erdmann on the classification of group blocks of tame representation type (see [4).

Later, the approach of 3 was applied to several families of algebras of quaternion type. Namely, in [5], the Hochschild cohomology algebras were calculated for a family of local algebras of quaternion type, and in [6, 7], the algebra $\operatorname{HH}^{*}(R)$ was described for the family $Q(2 \mathcal{B})_{1}$ over an algebraically closed field of characteristic 2 . We notice that bimodule resolutions for algebras of quaternion type were constructed also in 8 . Moreover, the approach of [3] was used for the calculation of the algebra $\operatorname{HH}^{*}(R)$ for Liu-Schulz algebras (see [9, 10]) and for the integer group ring $\mathbb{Z}\left[D_{4 m}\right]$ of the "even" dihedral group (i.e., of order $4 m$ ) [11. Note that an independent description of the algebra $\mathrm{HH}^{*}\left(\mathbb{Z}\left[D_{2 m}\right]\right)$ for all dihedral groups was obtained in [12, where the methods of the paper 1 ] were used.

Some particular results were obtained in the description of the algebra $\operatorname{HH}^{*}(R)$ for the so-called Möbius algebra (see [13, 15]), for a family of self-injective algebras of finite representation type with tree class $D_{n}$ (see [16]), and for group blocks of tame representation type that have one or three simple modules (see [17]).

2000 Mathematics Subject Classification. Primary 13D03.

Key words and phrases. Algebras of semidihedral type, Hochschild cohomology, semidihedral groups. Supported by RFBR, grant 06-01-00200. 
In the present paper, we use the technique of 3 to compute the Hochschild cohomology algebra for a family of local algebras of semidihedral type over an algebraically closed field of characteristic two. Since this family contains the group algebras of semidihedral groups (see 4]), this result can be employed for the description of the algebra $\operatorname{HH}^{*}(R)$ for such group algebras.

We outline the structure of the paper. In $\S 1$, we formulate the main result of the paper, Theorem 1.1. In it, for the family of local algebras of semidihedral type mentioned above, the algebra $\mathrm{HH}^{*}(R)$ is determined in terms of generators and relations. In $\S 2$, we build some bicomplex and use the spectral sequence of it to prove that the total complex of that bicomplex is the minimal projective resolution of the algebra $R$ viewed as a module over its enveloping algebra $\Lambda=R^{\mathrm{e}}=R \otimes_{K} R$. With the help of this resolution, in $\S 3$ we compute the groups $\mathrm{HH}^{n}(R)$. Finally, in $\S 4$ we pick a set of (homogeneous) generators for the algebra $\mathrm{HH}^{*}(R)$ and find relations satisfied by these generators.

It should be noted that the bimodule resolution mentioned above is constructed for algebras under consideration over the ground field of arbitrary characteristic.

\section{§1. Statement of the main Result}

Let $K$ be an algebraically closed field with arbitrary characteristic, $R$ a finite-dimensional $K$-algebra, $\Lambda=R^{\mathrm{e}}=R \otimes_{K} R^{\mathrm{op}}$ its enveloping algebra, and $\operatorname{HH}^{n}(R)=\operatorname{Ext}_{\Lambda}^{n}(R, R)$ the $n$th Hochschild cohomology group of $R$ (with coefficients in the $R$-bimodule $R$ ). The Hochschild cohomology algebra is the vector space

$$
\mathrm{HH}^{*}(R)=\bigoplus_{n \geq 0} \mathrm{HH}^{n}(R)=\bigoplus_{n \geq 0} \operatorname{Ext}_{\Lambda}^{n}(R, R)
$$

endowed with the $\smile$-product (see [18, §5], [19, Chapter XI], and [20]). The algebra $\mathrm{HH}^{*}(R)$ is a graded commutative algebra [20]; moreover, the $\smile$-product coincides with the Yoneda product on the Ext-algebra $\bigoplus_{n \geq 0} \operatorname{Ext}_{\Lambda}^{n}(R, R)$ of the $\Lambda$-module $R$; see 21, p. 120].

For any $k \in \mathbb{N}, k \geq 2$, we introduce the $K$-algebra $R_{k}=K\langle X, Y\rangle / I$, where $I$ is an ideal of the free algebra $K\langle X, Y\rangle ; I$ is generated by the elements

$$
X^{2}-Y(X Y)^{k-1}, Y^{2},(X Y)^{k}-(Y X)^{k}, X(Y X)^{k} .
$$

The images of $X$ and $Y$ under the canonical homomorphism from $K\langle X, Y\rangle$ into $R_{k}$ will be denoted by $x$ and $y$, respectively. The algebra $R_{k}$ is a symmetric local algebra of tame representation type [4, III.1.2]; moreover, the algebra $R_{k}$ is an algebra of semidihedral type in terms of [4, Chapter VIII]. If $G$ is the semidihedral group of order $2^{n}(n \geq 4)$ and char $K=2$, then the group algebra $K G$ is isomorphic to $R_{k}$ with $k=2^{n-2}$.

Below in this section, we assume that the ground field has characteristic two.

To describe the Hochschild cohomology algebra $\operatorname{HH}^{*}\left(R_{k}\right)$ for the algebras $R_{k}(k \geq 2)$, we construct several graded algebras. Put

$$
\mathcal{X}_{1}=\left\{p_{1}, p_{2}, p_{3}, p_{4}, u_{1}, u_{2}, u_{3}, v_{1}, v_{2}, v_{3}, w_{1}, w_{2}, t\right\} .
$$

We introduce a grading on the algebra $K\left[\mathcal{X}_{1}\right]$ so that

$$
\begin{array}{llrl}
\operatorname{deg} p_{1}=\operatorname{deg} p_{2}=\operatorname{deg} p_{3}=\operatorname{deg} p_{4}=0, & \operatorname{deg} u_{1}=\operatorname{deg} u_{2}=\operatorname{deg} u_{3}=1, \\
\operatorname{deg} v_{1}=\operatorname{deg} v_{2}=\operatorname{deg} v_{3}=2, & \operatorname{deg} w_{1}=\operatorname{deg} w_{2}=3, \operatorname{deg} t=4 .
\end{array}
$$

Then we define a graded $K$-algebra $\mathcal{A}_{1}=K\left[\mathcal{X}_{1}\right] / I_{1}$, where the ideal $I_{1}$ is generated by the following elements:

- of degree 0:

$$
\begin{gathered}
p_{1}^{k}, p_{2}^{2}, p_{3}^{2}, p_{4}^{2}, \\
p_{i} p_{j} \text { for } 1 \leq i<j \leq 4
\end{gathered}
$$


— of degree 1:

$$
\begin{gathered}
p_{1} u_{2}, p_{1} u_{1}+p_{3} u_{2}, p_{3} u_{1}+p_{2} u_{2} \\
p_{3} u_{3}, p_{4} u_{3}, p_{4} u_{1}+p_{2} u_{3}, p_{2} u_{2}+p_{1}^{k-1} u_{3}
\end{gathered}
$$

— of degree 2 :

$$
\begin{gathered}
p_{3} v_{1}, p_{4} v_{1}, p_{1}^{k-1} v_{2}, p_{3} v_{2}, p_{4} v_{2}, p_{1} v_{1}+p_{2} v_{2}, \\
p_{2} v_{1}+p_{4} u_{1}^{2}, \\
p_{1} v_{3}, p_{2} v_{3}, p_{4} v_{3}, \\
u_{1} u_{2}, u_{2} u_{3}, u_{3}^{2}, p_{2} v_{2}+p_{3} v_{3}, p_{2} v_{2}+p_{4} u_{2}^{2}
\end{gathered}
$$

— of degree 3 :

$$
\begin{gathered}
p_{3} w_{2}, p_{4} w_{2}, \\
u_{2}^{3}, u_{2} v_{2}, p_{1} w_{1}+u_{1} v_{2}, p_{3} w_{1}+u_{2} v_{1}, p_{4} w_{1}+p_{2} w_{2}, \\
u_{3} v_{3}, p_{3} w_{1}+u_{1} v_{3}, u_{1} v_{3}+p_{1}^{k-2} u_{3} v_{2}, p_{1} w_{1}+u_{2} v_{3}, \\
p_{4} w_{1}+u_{3} v_{1}, p_{1} w_{2}+u_{3} v_{2}, u_{1}^{2} u_{3}+u_{1} v_{1}+p_{2} w_{1}
\end{gathered}
$$

- of degree 4:

$$
\begin{gathered}
v_{1}^{2}, v_{1} v_{2}, v_{2}^{2}+p_{1}^{2} t \\
v_{1} v_{3}, v_{2} v_{3}, v_{3}^{2}, \\
u_{2} w_{1}, u_{2} w_{2}, u_{3} w_{2}, u_{1} w_{2}+u_{3} w_{1}
\end{gathered}
$$

— of degree 5:

$$
\begin{gathered}
v_{2} w_{1}+p_{3} u_{2} t \\
v_{3} w_{2}, v_{2} w_{2}+p_{1} u_{3} t, v_{1} w_{2}+p_{4} u_{1} t, v_{3} w_{1}+p_{3} u_{1} t \\
u_{1} u_{3} w_{1}+v_{1} w_{1}+p_{2} u_{1} t+\delta_{k, 2} p_{4} u_{2} t
\end{gathered}
$$

- of degree 6:

$$
\begin{aligned}
& w_{2}^{2}, w_{1}^{2}+u_{1}^{2} t, \\
& w_{1} w_{2}+u_{1} u_{3} t
\end{aligned}
$$

here, $\delta_{i, j}$ is the Kronecker delta. Moreover, we induce a grading on the algebra $\mathcal{A}_{1}$ from that on $K\left[\mathcal{X}_{1}\right]$.

Next, we consider the set

$$
\mathcal{X}_{2}=\left(\mathcal{X}_{1} \backslash\left\{u_{3}, v_{3}\right\}\right) \cup\left\{u_{3}^{\prime}\right\}
$$

with $\mathcal{X}_{1}$ as in (1.1). A grading on the algebra $\mathcal{K}\left[\mathcal{X}_{2}\right]$ is introduced by the requirement that the relations (1.2) be true for the elements of the set $\mathcal{X}_{1} \backslash\left\{u_{3}, v_{3}\right\}$, and $\operatorname{deg} u_{3}^{\prime}=1$. Then we introduce the algebra $\mathcal{A}_{2}=K\left[\mathcal{X}_{2}\right] / I_{2}$, where the ideal $I_{2}$ is generated by the generators of the ideal $I_{1}$ in (1.3), (1.4), (1.6), (1.7), (1.10), (1.11), (1.14), (1.16), (1.19), 
and by the elements

$$
\begin{gathered}
p_{2} u_{2}+p_{1}^{k-1} u_{3}^{\prime}, p_{4} u_{2}+p_{3} u_{3}^{\prime}, p_{2} u_{3}^{\prime}, p_{4} u_{3}^{\prime} ; \\
p_{2} v_{2}+p_{3} u_{2} u_{3}^{\prime}, u_{1} u_{2}+p_{1}^{k-2} v_{2},\left(u_{3}^{\prime}\right)^{2}, \\
p_{1} w_{1}+u_{2}^{2} u_{3}^{\prime}, p_{3} w_{1}+p_{1}^{k-1} w_{2}, p_{4} w_{1}+u_{3}^{\prime} v_{1}, \\
p_{1} w_{2}+u_{3}^{\prime} v_{2}, p_{2} w_{1}+u_{1}^{2} u_{3}^{\prime} ; \\
u_{2} w_{1}+p_{1}^{k-1} t, u_{2} w_{2}+p_{3} t, u_{3}^{\prime} w_{1}+p_{2} t, u_{3}^{\prime} w_{2}+p_{4} t \\
v_{1} w_{2}, v_{2} w_{2}+p_{1} u_{3}^{\prime} t, u_{1}^{2} w_{2}+v_{1} w_{1}, \\
w_{1} w_{2}+v_{1} t .
\end{gathered}
$$

Since the ideal $I_{2}$ is homogeneous, the grading on the algebra $K\left[\mathcal{X}_{2}\right]$ induces a grading on $\mathcal{A}_{2}$.

Theorem 1.1. Let $R=R_{k}$ with $k \in \mathbb{N} \backslash\{1\}$.

1) If $k$ is even, then the Hochschild cohomology algebra $\mathrm{HH}^{*}(R)$ is isomorphic, as a graded algebra, to $\mathcal{A}_{1}$.

2) If $k$ is odd, then $\mathrm{HH}^{*}(R) \simeq \mathcal{A}_{2}$ as graded $K$-algebras.

This description of the Hochschild cohomology algebra immediately implies the following statement.

Corollary 1.2. Let $G$ be the semidihedral group of order $2^{n} \quad(n \geq 4)$, and let $K$ be an algebraically closed field of characteristic 2 . Then, with $k=2^{n-2}$, we have $\operatorname{HH}^{*}(K[G]) \simeq$ $\mathcal{A}_{1}$.

In the proof of Theorem 1.1 we compute also the dimensions of the groups $\mathrm{HH}^{n}(R)$. Since these results are of independent interest, we collect them in the following separate statement.

Proposition 1.3. Let $R=R_{k}$. Then we have

$$
\operatorname{dim}_{K} \mathrm{HH}^{n}(R)= \begin{cases}k+3+8\left[\frac{n}{4}\right] & \text { if } n \equiv 0(\bmod 4), \\ k+6+8\left[\frac{n}{4}\right] & \text { if } n \equiv 1(\bmod 4), \\ k+7+8\left[\frac{n}{4}\right] & \text { if } n \equiv 2(\bmod 4), \\ k+8+8\left[\frac{n}{4}\right] & \text { if } n \equiv 3(\bmod 4) .\end{cases}
$$

Remark 1.4. Proposition 1.3 extends the corresponding result on the group algebras of the semidihedral groups, obtained in [17, to the entire family of algebras $R_{k}$.

\section{§2. Resolution}

Let $R=R_{k}$ be the algebra defined in $\$ 1$ The algebra $R$ admits the following set as a $K$-basis:

$$
\begin{aligned}
\mathcal{B}_{R}=\left\{(x y)^{i} \mid 0 \leq i \leq k\right\} & \cup\left\{(y x)^{i} \mid 1 \leq i \leq k-1\right\} \\
& \cup\left\{x(y x)^{i} \mid 0 \leq i \leq k-1\right\} \cup\left\{y(x y)^{i} \mid 0 \leq i \leq k-1\right\},
\end{aligned}
$$

which consists of all nonzero paths of the quiver of $R$ (this quiver contains one vertex and two loops $x$ and $y$ ). The set $\mathcal{B}_{R}$ is called the standard basis of $R$. In its turn, the enveloping algebra $\Lambda$ of $R$ has a $K$-basis consisting of elements of the form

$$
u \otimes v \text { with } u, v \in \mathcal{B}_{R} .
$$

This basis of $\Lambda$ will be denoted by $\mathcal{B}_{\Lambda}$; we also call it the standard basis. 
We introduce the following grading on $\Lambda$ : for an element of the form (2.1), we define its degree as the sum of the lengths of the paths $u$ and $v$, and then we put $\Lambda=\sum_{n>0} \Lambda_{n}$, where $\Lambda_{n}$ is the $K$-vector space spanned by the elements (2.1) with degree $n$.

Multiplication from the right by an element $\lambda \in \Lambda$ induces an endomorphism $\lambda^{*}$ of the left $\Lambda$-module $\Lambda$. In the sequel, this homomorphism will often be denoted simply by $\lambda$.

We consider the following elements of the algebra $\Lambda=R^{\mathrm{e}}$ :

$$
\begin{aligned}
Y^{-} & =y \otimes 1-1 \otimes y, \quad Y^{+}=y \otimes 1+1 \otimes y, \\
X^{-} & =x \otimes 1-1 \otimes x, \\
\varphi_{1} & =\sum_{i=0}^{k-1}(y x)^{i} \otimes(x y)^{k-1-i}, \\
\varphi_{2} & =-x \otimes 1-1 \otimes x+\sum_{i=0}^{k-2} y(x y)^{i} \otimes y(x y)^{k-2-i}, \\
\rho^{-} & =x(y x)^{k-1} \otimes 1-1 \otimes x(y x)^{k-1}, \\
\rho^{+} & =x(y x)^{k-1} \otimes 1+1 \otimes x(y x)^{k-1}, \\
\psi & =(y x)^{k-1} \otimes(x y)^{k-1}, \\
\tau & =Y^{+} \cdot X^{-}, \quad \sigma=X^{+} \cdot \varphi_{1} .
\end{aligned}
$$

Furthermore, we define the homomorphisms

$$
\begin{gathered}
\Phi=\left(\begin{array}{l}
\varphi_{1} \\
\varphi_{2}
\end{array}\right): \Lambda \rightarrow \Lambda^{2}, \Psi=\left(\begin{array}{ll}
\rho^{+} & \psi
\end{array}\right): \Lambda^{2} \rightarrow \Lambda, \\
\Upsilon=\left(\begin{array}{c}
Y^{+} \\
0
\end{array}\right): \Lambda \rightarrow \Lambda^{2} .
\end{gathered}
$$

In the category of (left) $\Lambda$-modules we construct the following bicomplex $\mathcal{B}_{\text {.. lying in }}$ the first quadrant of the plane (i.e., its rows and columns are numbered by $0,1,2, \ldots$ ):

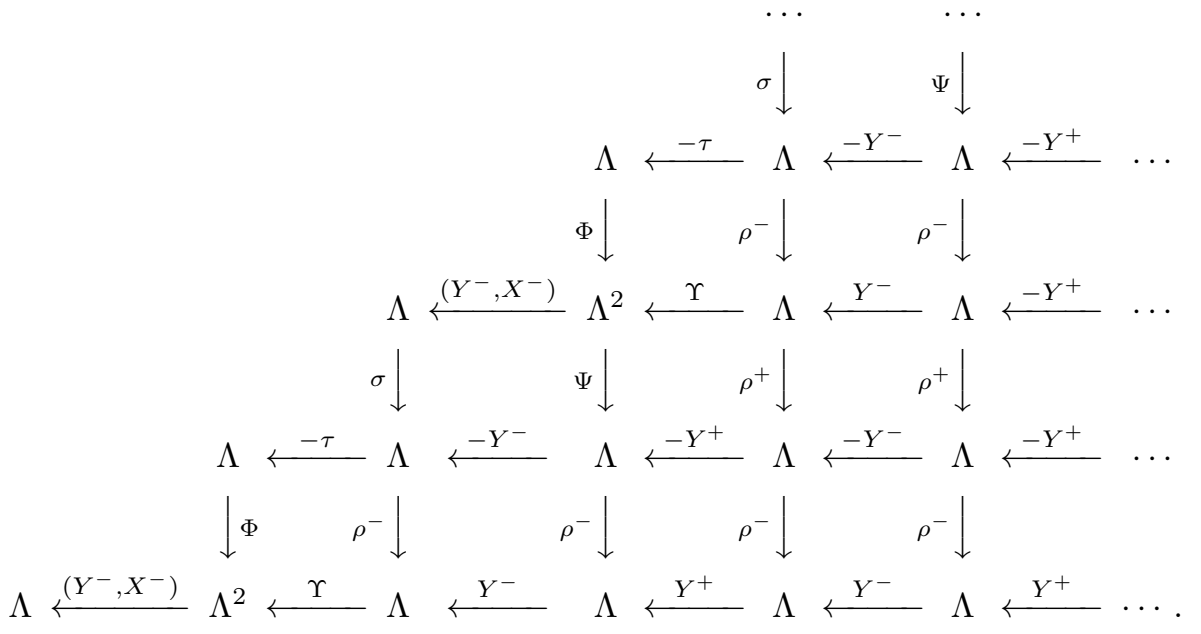

Theorem 2.1. The total complex $Q_{\bullet}=\operatorname{Tot}\left(\mathcal{B}_{\bullet}\right)$ of the bicomplex (2.10) is a minimal projective resolution of the $\Lambda$-module $R$.

Remark 2.2. Direct inspection shows that, indeed, the diagram (2.10) represents a bicomplex; i.e., its rows and columns are differential sequences and all squares are anticommutative. 
Remark 2.3. For the role of an augmentation map $\mu: Q_{0}=\Lambda \rightarrow R$, we take the canonical map induced by multiplication in $R: \mu(r \otimes s)=r s$. Then the exactness of the corresponding sequence at the member $Q_{0}$, that is, the relation $\operatorname{Im} d_{0}^{Q}=\operatorname{Ker} \mu$, is a well-known fact.

We shall use several auxiliary statements in the proof of Theorem 2.1

Lemma 2.4. Let $Y^{ \pm}: \Lambda \rightarrow \Lambda$ be a homomorphism of multiplication (from the right) by the corresponding element (see (2.2)). Then

$$
\operatorname{Ker} Y^{-}=\operatorname{Im} Y^{+}, \quad \operatorname{Ker} Y^{+}=\operatorname{Im} Y^{-} .
$$

Proof. We only prove the first relation. The second can be proved similarly. It is clear that $\operatorname{Im} Y^{+} \subset \operatorname{Ker} Y^{-}$.

Let $\lambda \in \operatorname{Ker} Y^{-}$, and let

$$
\lambda=\sum_{u, v \in \mathcal{B}_{R}} \lambda(u, v) u \otimes v
$$

be the expansion of this element with respect to the standard basis of the algebra $\Lambda$. Subtracting the element

$$
\left(\sum_{v \in \mathcal{B}_{R}}\left(\sum_{i=0}^{k-1} \lambda\left(y(x y)^{i}, v\right)(y x)^{i} \otimes v+\sum_{i=1}^{k} \lambda\left((x y)^{i}, v\right) x(y x)^{i-1} \otimes v\right)\right) \cdot Y^{+} \in \operatorname{Ker} Y^{-},
$$

from $\lambda$, we obtain an element whose expansion does not involve summands that are multiples of a basic element $u \otimes v$ such that $u$ is a path with the first letter $y$. Hence, we may assume that the initial element $\lambda$ has such property. Then the relation $\lambda \cdot y \otimes 1=$ $\lambda \cdot 1 \otimes y$ implies that

$$
\begin{aligned}
\sum_{v \in \mathcal{B}_{R}} & \left(\lambda(1, v) y \otimes v+\sum_{i=0}^{k-1} \lambda\left(x(y x)^{i}, v\right)(x y)^{i+1} \otimes v+\sum_{i=1}^{k-1} \lambda\left((y x)^{i}, v\right) y(x y)^{i} \otimes v\right) \\
= & \sum_{v \in \mathcal{B}_{R}}\left(\lambda(1, v) 1 \otimes y v+\sum_{i=0}^{k-1} \lambda\left(x(y x)^{i}, v\right) x(y x)^{i} \otimes y v+\sum_{i=1}^{k-1} \lambda\left((y x)^{i}, v\right)(x y)^{i} \otimes y v\right) .
\end{aligned}
$$

Here the left-hand side is equal to zero, because the right-hand side in (2.11) involves no basis vectors in $\mathcal{B}_{\Lambda}$ that are included in the linear combination presented on the lefthand side of (2.11). Consequently, all coefficients $\lambda(u, v)$ on the left in (2.11) are zero, whence $\lambda=0$.

The following lemma implies the exactness of the above sequence at the member $Q_{1}$.

Lemma 2.5. $\operatorname{Ker} d_{0}^{Q} \subset \operatorname{Im} d_{1}^{Q}$.

Proof. The proof repeats word for word the proof of Lemma 2.4 in [5]. Indeed, we note that the matrix of the differential $d_{1}$ is congruent to the diagonal matrix $\operatorname{diag}\left(Y^{+}, X^{+}\right)$ modulo $(\operatorname{Rad} \Lambda)^{2}$, but the matrix of the differential $d_{1}$ in [5, (2.3)] is congruent to the same diagonal matrix (again modulo $\left.(\operatorname{Rad} \Lambda)^{2}\right)$.

Lemma 2.6. If $\left(\lambda_{1}, \lambda_{2}\right) \in \operatorname{Ker} d_{1}^{Q}$, then $\lambda_{2} \in \operatorname{Im} \tau^{*}$.

Proof. The relation $d_{1}^{Q}\left(\lambda_{1}, \lambda_{2}\right)=0$ is equivalent to the system

$$
\begin{aligned}
& \lambda_{1} Y^{+}+\lambda_{2} \varphi_{1}=0, \\
& \lambda_{2} \varphi_{2}=0 .
\end{aligned}
$$


By Lemma 2.4 the first equation in (2.12) is satisfied by an element $\lambda$ exactly when we have $\lambda_{2} \cdot \Xi=0$ with

$$
\Xi:=\varphi_{1} \cdot Y^{-}=\sum_{j=0}^{k-1}(y x)^{j} y \otimes(x y)^{k-1-j}-\sum_{j=0}^{k-1}(y x)^{j} \otimes y(x y)^{k-1-j} .
$$

Hence, we need to check that if $\lambda \in \Lambda$ satisfies the relations

$$
\begin{aligned}
\lambda \cdot \Xi & =0, \\
\lambda \cdot \varphi_{2} & =0,
\end{aligned}
$$

then $\lambda \in \operatorname{Im} \tau^{*}$. It is useful to observe that the reverse implication is also valid (this is verified by direct calculation): if $\lambda \in \operatorname{Im} \tau^{*}$, then $\lambda$ satisfies (2.13).

Assume that $\lambda$ satisfies (2.13). Let $\lambda^{(t)}$ be the homogeneous component in $\lambda$ of degree t. A direct calculation gives $\lambda^{(0)}=0=\lambda^{(1)}$.

We fix $t \geq 2$ such that $\lambda^{(i)}=0$ for all $i<t$. We shall modify the element $\lambda$ successively by adding elements in $\operatorname{Im} \tau^{*}$ so as to get an element in which all homogeneous components of degrees $i \leq t$ are zero. After finitely many steps, we replace the initial $\lambda$ by the zero element, whence the desired inclusion will follow.

Step 1. First we consider the case where $t=2 \ell$ with $1 \leq \ell \leq k-1$. We represent the homogeneous component $\lambda^{(t)}$ as a linear combination of the standard basis elements in $\Lambda$ (with coefficients $\alpha_{i}, \ldots, f_{i}$ in $K$ ):

$$
\begin{aligned}
\lambda^{(t)}= & \sum_{i=0}^{\ell} \alpha_{i}(x y)^{i} \otimes(x y)^{\ell-i}+\sum_{i=0}^{\ell-1} \beta_{i}(x y)^{i} \otimes(y x)^{\ell-i} \\
& +\sum_{i=1}^{\ell} \gamma_{i}(y x)^{i} \otimes(x y)^{\ell-i}+\sum_{i=1}^{\ell-1} \varepsilon_{i}(y x)^{i} \otimes(y x)^{\ell-i} \\
& +\sum_{i=0}^{\ell-1} a_{i} x(y x)^{i} \otimes x(y x)^{\ell-1-i}+\sum_{i=0}^{\ell-1} b_{i} x(y x)^{i} \otimes y(x y)^{\ell-1-i} \\
& +\sum_{i=0}^{\ell-1} c_{i} y(x y)^{i} \otimes x(y x)^{\ell-1-i}+\sum_{i=0}^{\ell-1} f_{i} y(x y)^{i} \otimes y(x y)^{\ell-1-i} .
\end{aligned}
$$

Substituting (2.14) in the first relation in (2.13), we obtain the following equations for the scalar coefficients in (2.14):

$$
\begin{aligned}
\alpha_{0}+\sum_{i=1}^{\ell} \gamma_{i} & =0=\alpha_{0}+\sum_{i=1}^{\ell-1} \gamma_{i}-c_{\ell-1}, \\
b_{0}-\sum_{i=1}^{\ell} \gamma_{i} & =0 \\
\sum_{i=0}^{\ell-1} a_{i} & =0 \\
\alpha_{0}=\beta_{0}=\alpha_{\ell} & =0 .
\end{aligned}
$$


Similarly, the second relation in (2.13) is equivalent to the following:

$$
\begin{aligned}
\alpha_{i}+b_{i} & =0 & & \text { for } 0 \leq i \leq \ell-1, \\
c_{i-1}+\varepsilon_{i} & =0 & & \text { for } 1 \leq i \leq \ell-1, \\
\beta_{i}=f_{i} & =0 & & \text { for } 0 \leq i \leq \ell-1, \\
\alpha_{\ell}=\gamma_{\ell}=c_{\ell-1} & =0 . & &
\end{aligned}
$$

Since $\alpha_{0}=0$, we also have $b_{0}=0$ (see (2.18) and (2.19) $)$. Replacing $\lambda$ by the element

$$
\lambda+\left(\sum_{i=1}^{\ell-1} \alpha_{i}(x y)^{i} \otimes(x y)^{\ell-1-i}\right) \cdot \tau,
$$

we obtain an element such that in the canonical expansion of it the coefficients of $(x y)^{i} \otimes$ $(x y)^{\ell-i}(1 \leq i \leq \ell-1)$ are zero. Hence, we may assume that the initial element $\lambda$ has the property that $\alpha_{i}=0$ for $1 \leq i \leq \ell-1$. By (2.19), we also have $b_{i}=0$ for $1 \leq i \leq \ell-1$.

Similarly, replacing $\lambda$ by the element

$$
\lambda-\left(\sum_{i=1}^{\ell-1} \varepsilon_{i} y(x y)^{i-1} \otimes x(y x)^{\ell-1-i}\right) \cdot \tau,
$$

we may assume that $\varepsilon_{i}=0$ for all $1 \leq i \leq \ell-1$. Then, by (2.20), also $c_{i}=0$ for $0 \leq i \leq \ell-2$. Next, if there exists an index $i \leq \ell-1$ with $\gamma_{i} \neq 0$, then we pick the smallest $j$ such that $\gamma_{j} \neq 0$. Adding the element

$$
\gamma_{j}(y x)^{j} \otimes(x y)^{\ell-1-j} \cdot \tau
$$

to $\lambda$, we obtain an element for which the coefficients of $(y x)^{i} \otimes(x y)^{\ell-i}$ with $i \leq j$ are zero (observe that $j<\ell-1$ ). Repeating this procedure, we obtain an element for which the coefficients of $(y x)^{i} \otimes(x y)^{\ell-i}$ with $i \leq \ell-1$ are zero. Hence, we may assume that in the initial $\lambda$ we have $\gamma_{i}=0$ for $1 \leq i \leq \ell-1$. If there exist nonzero coefficients $a_{i}(0 \leq i \leq$ $\ell-1$ ), then a similar procedure (invoking elements of the form $x(y x)^{j-1} \otimes x(y x)^{\ell-1-j} \cdot \tau$ ) results in an element for which the coefficients of $x(y x)^{i} \otimes x(y x)^{\ell-1-i}$ for $0 \leq i \leq \ell-2$ are zero. Hence, we may assume that, in the initial element $\lambda$, we have $a_{i}=0$ for $0 \leq i \leq \ell-2$, and, by (2.17),$a_{\ell-1}=0$. Consequently, we have $\lambda^{(t)}=0$ in the resulting element $\lambda$.

Step 2. Now we put $t=2 \ell+1$ with $1 \leq \ell<k-1$ and write

$$
\begin{aligned}
\lambda^{(t)}= & \sum_{i=0}^{\ell} \alpha_{i}(x y)^{i} \otimes x(x y)^{\ell-i}+\sum_{i=0}^{\ell} \beta_{i}(x y)^{i} \otimes y(x y)^{\ell-i} \\
& +\sum_{i=1}^{\ell} \gamma_{i}(y x)^{i} \otimes x(y x)^{\ell-i}+\sum_{i=1}^{\ell} \varepsilon_{i}(y x)^{i} \otimes y(x y)^{\ell-i} \\
& +\sum_{i=0}^{\ell} a_{i} x(y x)^{i} \otimes(x y)^{\ell-i}+\sum_{i=0}^{\ell-1} b_{i} x(y x)^{i} \otimes(y x)^{\ell-i} \\
& +\sum_{i=0}^{\ell} c_{i} y(x y)^{i} \otimes(x y)^{\ell-i}+\sum_{i=0}^{\ell-1} f_{i} y(x y)^{i} \otimes(y x)^{\ell-i},
\end{aligned}
$$


where the coefficients $\alpha_{i}, \ldots, f_{i}$ lie in $K$. Substitution of (2.23) in the two relations in (2.13) gives the following equations:

$$
\begin{aligned}
& \alpha_{0}+\sum_{i=1}^{\ell} \gamma_{i}=0, \\
& b_{0}-\sum_{i=1}^{\ell} \gamma_{i}=\sum_{i=0}^{\ell} a_{i}=0 \text {, } \\
& \alpha_{i}+b_{i}=0 \\
& \text { for } 0 \leq i \leq \ell-1 \text {, } \\
& c_{i-1}+\varepsilon_{i}=0 \\
& \text { for } 1 \leq i \leq \ell \text {, } \\
& \beta_{i}=0 \\
& \text { for } 0 \leq i \leq \ell \text {, } \\
& f_{i}=0 \\
& \text { for } 0 \leq i \leq \ell-1 \text {, } \\
& \alpha_{\ell}+a_{\ell}=c_{\ell}=\alpha_{\ell}=\gamma_{\ell}=a_{0}=b_{0}=0 \text {. }
\end{aligned}
$$

Adding the element

$$
\left(\sum_{i=0}^{\ell-1} \alpha_{i}(x y)^{i} \otimes x(y x)^{\ell-1-i}\right) \cdot \tau
$$

to $\lambda$, we obtain an element for which the coefficients of $(x y)^{i} \otimes x(y x)^{\ell-i}$ with $0 \leq i \leq \ell-1$ are zero. Hence, we may assume that, in the initial $\lambda$, we have $\alpha_{i}=0$ for all $0 \leq i \leq \ell-1$, and then also $b_{i}=0$ for $0 \leq i \leq \ell-1$. Similarly, using elements of the form

$$
y(x y)^{i-1} \otimes(x y)^{\ell-i} \cdot \tau, \quad 1 \leq i \leq \ell,
$$

we may assume that $\varepsilon_{i}=0$ for $1 \leq i \leq \ell$, and then $c_{i}=0$ for $0 \leq i \leq \ell-1$. If there exists an index $i \leq \ell-1$ with $\gamma_{i} \neq 0$, we pick the smallest index $j$ such that $\gamma_{j} \neq 0$ (note that $j<\ell-1)$. Adding the element

$$
\gamma_{j}(y x)^{j} \otimes x(y x)^{\ell-1-j} \cdot \tau
$$

to $\lambda$, we obtain an element for which the coefficients of $(y x)^{i} \otimes x(y x)^{\ell-i}$ with $i \leq j$ are zero. Repeating this process, we obtain an element for which the coefficients of $(y x)^{i} \otimes x(y x)^{\ell-i}$ with $i \leq \ell-1$ are zero. Hence, we may assume that in the initial element $\lambda$ we have $\gamma_{i}=0$ for $1 \leq i \leq \ell-1$. Similarly, using elements of the form

$$
x(y x)^{j} \otimes(x y)^{\ell-1-j} \cdot \tau,
$$

we may assume that in the initial $\lambda$ we have $a_{i}=0$ for $0 \leq i \leq \ell$, and then we deduce that $\lambda^{(t)}=0$.

Step 3. Next, we consider the case where $t=2 k-1$. Again, the homogeneous component $\lambda^{(2 k-1)}$ can be presented in the form (2.23) (by putting $\ell=k-1$ ). As at Step 2, we obtain the same relations (2.24) -(2.30) except the first, which must be replaced by the following relation:

$$
\alpha_{0}+\sum_{i=1}^{\ell} \gamma_{i}-c_{k-1}=0
$$

But the second equation in (2.13) shows that $c_{k-1}=0$, so that we can apply the arguments of Step 2, obtaining the desired statement for the case under consideration. 
Step 4. Now we put $t=2 k$. We present the homogeneous component $\lambda^{(2 k)}$ in the form

$$
\begin{aligned}
\lambda^{(2 k)}= & \sum_{i=0}^{k} \alpha_{i}(x y)^{i} \otimes(x y)^{k-i}+\sum_{i=1}^{k-1} \beta_{i}(x y)^{i} \otimes(y x)^{k-i} \\
& +\sum_{i=1}^{k-1} \gamma_{i}(y x)^{i} \otimes(x y)^{k-i}+\sum_{i=1}^{k-1} \varepsilon_{i}(y x)^{i} \otimes(y x)^{k-i} \\
& +\sum_{i=0}^{k-1} a_{i} x(y x)^{i} \otimes x(y x)^{k-1-i}+\sum_{i=0}^{k-1} b_{i} x(y x)^{i} \otimes y(x y)^{k-1-i} \\
& +\sum_{i=0}^{k-1} c_{i} y(x y)^{i} \otimes x(y x)^{k-1-i}+\sum_{i=0}^{k-1} f_{i} y(x y)^{i} \otimes y(x y)^{k-1-i} .
\end{aligned}
$$

Substituting (2.31) in (2.13) leads to the relations

$$
\begin{aligned}
& \alpha_{0}+\sum_{i=1}^{k-1} \gamma_{i}-c_{k-1}=0, \quad b_{0}-\sum_{i=1}^{k-1} \gamma_{i}-\alpha_{k}=0, \quad \sum_{i=0}^{k-1} a_{i}=0, \\
& \alpha_{i}+b_{i}=0 \quad \text { for } 0 \leq i \leq k-1 \text {, } \\
& c_{i-1}+\varepsilon_{i}=0 \quad \text { for } 1 \leq i \leq k-1 \text {, } \\
& \beta_{i}=0 \quad \text { for } 1 \leq i \leq k-1 \text {, } \\
& f_{i}=0 \quad \text { for } 0 \leq i \leq k-1 \text {, } \\
& \alpha_{k}+c_{k-1}=0 \text {. }
\end{aligned}
$$

Applying reductions similar to that at Step 1, we put successively the following restrictions on $\lambda$ :

a) $\alpha_{i}=0$ for $0 \leq i \leq k-1$ (we get this by using the elements $(x y)^{i} \otimes(x y)^{k-1-i} \cdot \tau$ ); then we also have $b_{i}=0$ for $0 \leq i \leq k-1$;

b) $\alpha_{k}=0=c_{k-1}$ (here, we use the element $(y x)^{k-1} \otimes 1 \cdot \tau$ );

c) $\varepsilon_{i}=0$ for $1 \leq i \leq k-1$ (here, we use the elements $\left.y(x y)^{i-1} \otimes x(y x)^{k-1-i} \cdot \tau\right)$; then we also have $c_{i}=0$ for $0 \leq i \leq k-2$;

d) $\gamma_{i}=0$ for $1 \leq i \leq k-1$ (we use the elements $\left.(y x)^{i} \otimes(x y)^{k-1-i} \cdot \tau\right)$;

e) $a_{i}=0$ for $0 \leq i \leq k-1$ (we use the elements $x(y x)^{i} \otimes x(y x)^{k-2-i} \cdot \tau$ ). Clearly, we obtain $\lambda^{(2 k)}=0$.

Step 5. Put $t=2 \ell+1$ with $k \leq \ell \leq 2 k-1$. Denote $\bar{\ell}=\ell-k$. We write the homogeneous component $\lambda^{(t)}$ in the form

$$
\begin{aligned}
\lambda^{(t)}= & \sum_{i=\bar{\ell}+1}^{k} \alpha_{i}(x y)^{i} \otimes x(y x)^{\ell-i}+\sum_{i=\bar{\ell}+1}^{k} \beta_{i}(x y)^{i} \otimes y(x y)^{\ell-i} \\
& +\sum_{i=\bar{\ell}+1}^{k-1} \gamma_{i}(y x)^{i} \otimes x(y x)^{\ell-i}+\sum_{i=\bar{\ell}+1}^{k-1} \varepsilon_{i}(y x)^{i} \otimes y(x y)^{\ell-i} \\
& +\sum_{i=\bar{\ell}}^{k-1} a_{i} x(y x)^{i} \otimes(x y)^{\ell-i}+\sum_{i=\bar{\ell}+1}^{k-1} b_{i} x(y x)^{i} \otimes(y x)^{\ell-i} \\
& +\sum_{i=\bar{\ell}}^{k-1} c_{i} y(x y)^{i} \otimes(x y)^{\ell-i}+\sum_{i=\bar{\ell}+1}^{k-1} f_{i} y(x y)^{i} \otimes(y x)^{\ell-i} .
\end{aligned}
$$


If $\ell>k$, then $\lambda^{(t)} \cdot \Xi=0$; hence, we only need to analyze the second equation in (2.13). Substitution of (2.32) in (2.13) gives the following relations:

$$
\begin{aligned}
\alpha_{i}+b_{i} & =0 & & \text { for } \bar{\ell}+1 \leq i \leq k-1, \\
c_{i-1}+\varepsilon_{i} & =0 & & \text { for } \bar{\ell}+1 \leq i \leq k-1, \\
\beta_{i} & =0 & & \text { for } \bar{\ell}+1 \leq i \leq k-1, \\
f_{i} & =0 & & \text { for } \bar{\ell}+1 \leq i \leq k-1, \\
\beta_{k}+c_{k-1} & =0 . & &
\end{aligned}
$$

(Observe that for $\ell=2 k-1$ this list of relations reduces to relation (2.37) only.) If $\ell=k$, then we join one additional relation to the above relations, namely

$$
\alpha_{k}+\sum_{i=1}^{k-1} \gamma_{i}+\sum_{i=0}^{k-1} a_{i}=0 .
$$

Now we can argue as before to prove the desired statement.

Step 6. Put $t=2 \ell$ with $k<\ell \leq 2 k$ and denote $\bar{\ell}=\ell-k$. Then the homogeneous component $\lambda^{(t)}$ takes the form

$$
\begin{aligned}
\lambda^{(t)}= & \sum_{i=\bar{\ell}}^{k} \alpha_{i}(x y)^{i} \otimes(x y)^{\ell-i}+\sum_{i=\bar{\ell}+1}^{k} \beta_{i}(x y)^{i} \otimes(y x)^{\ell-i} \\
& +\sum_{i=\bar{\ell}}^{k-1} \gamma_{i}(y x)^{i} \otimes(x y)^{\ell-i}+\sum_{i=\bar{\ell}+1}^{k-1} \varphi_{i}(y x)^{i} \otimes(y x)^{\ell-i} \\
& +\sum_{i=\bar{\ell}}^{k-1} a_{i} x(y x)^{i} \otimes x(y x)^{\ell-1-i}+\sum_{i=\bar{\ell}}^{k-1} b_{i} x(y x)^{i} \otimes y(x y)^{\ell-1-i} \\
& +\sum_{i=\bar{\ell}}^{k-1} c_{i} y(x y)^{i} \otimes x(y x)^{\ell-1-i}+\sum_{i=\bar{\ell}}^{k-1} f_{i} y(x y)^{i} \otimes y(x y)^{\ell-1-i} .
\end{aligned}
$$

Again we have $\lambda^{(t)} \cdot \Xi=0$; hence, we only need to analyze the second equation in (2.13). Substitution of (2.38) in (2.13) gives the following relations:

$$
\begin{aligned}
\alpha_{i}+b_{i} & =0 & & \text { for } \bar{\ell} \leq i \leq k-1, \\
c_{i-1}+\varepsilon_{i} & =0 & & \text { for } \bar{\ell}+1 \leq i \leq k-1, \\
\beta_{i} & =0 & & \text { for } \bar{\ell}+1 \leq i \leq k-1, \\
f_{i} & =0 & & \text { for } \bar{\ell} \leq i \leq k-1, \\
\beta_{k}+c_{k-1} & =0 . & &
\end{aligned}
$$

(If $\ell=2 k$, we have no relations.) Now, arguing as above, we obtain the desired statement with the help of suitable reductions of the element $\lambda$.

Lemma 2.7. $\operatorname{Ker}\left(\sigma Y^{+}\right)^{*} \subset \operatorname{Im} d_{0}^{Q}$.

Proof. Put

$$
\begin{aligned}
\Theta:=\sigma Y^{+}= & \sum_{j=0}^{k-1}(x y)^{j+1} \otimes(x y)^{k-1-j}+\sum_{j=0}^{k-1} y(x y)^{j} \otimes x(y x)^{k-1-j} \\
& +\sum_{j=0}^{k-1} x(y x)^{j} \otimes y(x y)^{k-1-j}+\sum_{j=0}^{k-1}(y x)^{j} \otimes(y x)^{k-j}
\end{aligned}
$$

Observe that $\operatorname{Im} d_{0}^{Q} \subset \operatorname{Ker} \Theta^{*}$. 
Assume that the element $\lambda \in \Lambda$ satisfies

$$
\lambda \cdot \Theta=0 .
$$

As in the proof of Lemma 2.6 we consider a nonzero component $\lambda^{(t)}$ of the element $\lambda$ with the smallest possible value of $t$. Clearly, $\lambda^{(0)}=0$. We shall change the element $\lambda$ by subsequently adding elements of $\operatorname{Im} d_{0}^{Q}=\operatorname{Im}\left(\left(Y^{-}\right)^{*},\left(X^{-}\right)^{*}\right)$ to $\lambda$, in order to obtain an element in which all homogeneous components of degree $i \leq t$ are zero.

Step 1. First, we consider the case where $t=2 \ell+1$ with $0 \leq \ell \leq k-1$. We write the homogeneous component $\lambda^{(t)}$ in the form (2.23) (with coefficients $\alpha_{i}, \ldots, f_{i}$ in $K$ ). Substitution of (2.23) in (2.39) gives the following relations:

$$
\begin{aligned}
& \sum_{i=0}^{\ell} \alpha_{i}+\sum_{i=0}^{\ell-1} b_{i}+a_{\ell}=0 \\
& \sum_{i=1}^{\ell} \varepsilon_{i}+\sum_{i=0}^{\ell} c_{i}+\beta_{0}=0 .
\end{aligned}
$$

Next, we put successively additional restrictions on $\lambda$.

a) We may assume that $\beta_{i}=0$ for $1 \leq i \leq \ell$. For this, we can replace $\lambda$ by an element of the form

$$
\lambda-\left(\sum_{i=1}^{\ell} \beta_{i} x(y x)^{i-1} \otimes y(x y)^{\ell-i}\right) \cdot Y^{-}
$$

in which the coefficients of $(x y)^{i} \otimes y(x y)^{\ell-i}, i=1, \ldots, \ell$, are zero.

b) We may assume that $\gamma_{i}=0$ for $1 \leq i \leq \ell$. For this, we can replace $\lambda$ by an element of the form

$$
\lambda+\left(\sum_{i=1}^{\ell} \gamma_{i}(y x)^{i} \otimes(y x)^{\ell-i}\right) \cdot X^{-} .
$$

c) We may assume that $f_{i}=0$ for $0 \leq i \leq \ell-1$. For this, we can replace $\lambda$ by an element of the form

$$
\lambda-\left(\sum_{i=0}^{\ell-1} f_{i}(y x)^{i} \otimes(y x)^{\ell-i}\right) \cdot Y^{-} .
$$

d) We may assume that $a_{i}=0$ for $0 \leq i \leq \ell-1$. For this, we can replace $\lambda$ by an element of the form

$$
\lambda+\left(\sum_{i=0}^{\ell-1} a_{i} x(y x)^{i} \otimes y(x y)^{\ell-1-i}\right) \cdot X^{-} .
$$

Note that, unlike the previous cases, some components $\lambda^{(j)}$ with $j>t$ can be changed under such a replacement.

e) We may assume that $\alpha_{i}=0$ for $0 \leq i \leq \ell, b_{i}=0$ for $0 \leq i \leq \ell-1$, and $a_{\ell}=0$. Indeed, we have the following easily verified relation, in which the right-hand 
side contains two sums of the corresponding elements in the expansion (2.23):

$$
\begin{aligned}
\Omega:= & \sum_{j=0}^{\ell-1}\left(\sum_{i=\ell-j}^{\ell} \alpha_{i}+\sum_{i=\ell-j}^{\ell-1} b_{i}\right) x(y x)^{\ell-1-j} \otimes x(y x)^{j} \cdot Y^{-} \\
& +\sum_{j=0}^{\ell-1}\left(\sum_{i=\ell-j}^{\ell} \alpha_{i}+\sum_{i=\ell-1-j}^{\ell-1} b_{i}\right)(x y)^{\ell-1-j} \otimes(y x)^{j+1} \cdot X^{-} \\
= & \sum_{j=0}^{\ell} \alpha_{j}(x y)^{j} \otimes x(y x)^{\ell-j}+\sum_{j=0}^{\ell-1} b_{i} x(y x)^{j} \otimes(y x)^{\ell-j}+a_{\ell} \cdot 1 \otimes x(y x)^{\ell} .
\end{aligned}
$$

Replacing $\lambda$ by $\lambda-\Omega$, we obtain an element for which the expansion in the standard basis contains the following group of two elements:

$$
\omega:=a_{\ell}\left(x(y x)^{\ell} \otimes 1-1 \otimes x(y x)^{\ell}\right) .
$$

Since $\mu(\omega)=0$, we have $\omega \in \operatorname{Im} d_{0}^{Q}$. Then we subtract $\omega$ from $\lambda$, obtaining an element for which the coefficient of the basis element $x(y x)^{\ell} \otimes 1$ is equal to zero.

f) We may assume that $\varepsilon_{i}=0$ for $0 \leq i \leq \ell-1, c_{i}=0$ for $1 \leq i \leq \ell$, and $\beta_{0}=0$. We consider the following identity, in which the right-hand side is the sum of the corresponding elements in the expansion (2.23):

$$
\begin{aligned}
\Omega_{1}:= & \sum_{j=0}^{\ell}\left(\sum_{i=\ell-j}^{\ell} c_{i}+\sum_{i=\ell+1-j}^{\ell} \varepsilon_{i}\right)(y x)^{\ell-j} \otimes(x y)^{j} \cdot Y^{-} \\
& +\sum_{j=0}^{\ell-1}\left(\sum_{i=\ell-j}^{\ell} c_{i}+\sum_{i=\ell-j}^{\ell} \varepsilon_{i}\right) y(x y)^{\ell-1-j} \otimes y(x y)^{j} \cdot X^{-} \\
= & \sum_{j=0}^{\ell} c_{j} y(x y)^{j} \otimes(x y)^{\ell-j}+\sum_{j=1}^{\ell} \varepsilon_{i}(y x)^{j} \otimes y(x y)^{\ell-j}+\beta_{0} \cdot 1 \otimes y(x y)^{\ell} .
\end{aligned}
$$

It remains to replace $\lambda$ by $\lambda-\Omega_{1}$.

Clearly, if $\lambda$ satisfies the assumptions of cases a) $-\mathrm{f})$, then $\lambda^{(t)}=0$.

Step 2. Next, we consider the case where $t=2 \ell$ with $1 \leq \ell \leq k-1$. We write the homogeneous component $\lambda^{(t)}$ in the form (2.14). Substituting this in (2.39), we obtain the following equations for the coefficients in (2.14):

$$
\begin{array}{r}
\sum_{i=0}^{\ell} \alpha_{i}+\sum_{i=0}^{\ell-1} b_{i}=0, \\
\sum_{i=1}^{\ell-1} \varepsilon_{i}+\sum_{i=0}^{\ell-1} c_{i}+\beta_{0}+\gamma_{\ell}=0 .
\end{array}
$$

As at Step 1, we successively put additional restrictions on $\lambda$.

a) We may assume that $\beta_{i}=0$ for $1 \leq i \leq \ell-1$. For this, we can replace $\lambda$ by an element of the form

$$
\lambda-\left(\sum_{i=1}^{\ell-1} \beta_{i}(x y)^{i-1} \otimes(y x)^{\ell-i}\right) \cdot Y^{-} .
$$

b) We may assume that $\gamma_{i}=0$ for $1 \leq i \leq \ell-1$. For this, we can replace $\lambda$ by an element of the form

$$
\lambda-\left(\sum_{i=1}^{\ell-1} \gamma_{i} y(x y)^{i-1} \otimes(x y)^{\ell-i}\right) \cdot X^{-} .
$$


c) We may assume that $f_{i}=0$ for $0 \leq i \leq \ell-1$. For this, we can replace $\lambda$ by an element of the form

$$
\lambda-\left(\sum_{i=0}^{\ell-1} f_{i}(y x)^{i} \otimes y(x y)^{\ell-1-i}\right) \cdot Y^{-} .
$$

d) We may assume that $a_{i}=0$ for $0 \leq i \leq \ell-1$. For this, we can replace $\lambda$ by an element of the form

$$
\lambda-\left(\sum_{i=0}^{\ell-1} a_{i}(x y)^{i} \otimes x(y x)^{\ell-1-i}\right) \cdot X^{-} .
$$

e) We may assume that $\alpha_{i}=0$ for $0 \leq i \leq \ell$ and that $b_{i}=0$ for $0 \leq i \leq \ell-1$. As at Step 1, for this we use the following identity, in which the right-hand side is the sum of the corresponding elements in (2.14):

$$
\begin{aligned}
\Omega^{\prime}:= & \sum_{j=0}^{\ell}\left(\sum_{i=\ell-j+1}^{\ell} \alpha_{i}+\sum_{i=\ell-j+1}^{\ell-1} b_{i}\right) x(y x)^{\ell-j} \otimes(x y)^{j-1} \cdot Y^{-} \\
& +\sum_{j=0}^{\ell}\left(\sum_{i=\ell-j+1}^{\ell} \alpha_{i}+\sum_{i=\ell-j}^{\ell-1} b_{i}\right)(x y)^{\ell-j} \otimes y(x y)^{j-1} \cdot X^{-} \\
= & \sum_{j=0}^{\ell} \alpha_{j}(x y)^{j} \otimes(x y)^{\ell-j}+\sum_{j=0}^{\ell-1} b_{i} x(y x)^{j} \otimes y(x y)^{\ell-j-1} .
\end{aligned}
$$

It remains to replace $\lambda$ by $\lambda-\Omega^{\prime}$.

f) Finally, we may assume that $\varepsilon_{i}=0$ for $1 \leq i \leq \ell-1, c_{i}=0$ for $0 \leq i \leq \ell-1$, and $\beta_{0}=\gamma_{\ell}=0$. Again, we use an identity similar to (2.40). To present this identity, it is convenient to introduce the following auxiliary notation:

$$
\varepsilon_{0}:=\beta_{0}, \varepsilon_{\ell}:=\gamma_{\ell} .
$$

Then we have

$$
\begin{aligned}
\Omega_{1}^{\prime}:= & \sum_{j=0}^{\ell}\left(\sum_{i=\ell-j}^{\ell-1} c_{i}+\sum_{i=\ell-j}^{\ell} \varepsilon_{i}\right) y(x y)^{\ell-1-j} \otimes(y x)^{j} \cdot X^{-} \\
& +\sum_{j=0}^{\ell-1}\left(\sum_{i=\ell-1-j}^{\ell-1} c_{i}+\sum_{i=\ell-j}^{\ell} \varepsilon_{i}\right)(y x)^{\ell-1-j} \otimes x(y x)^{j} \cdot Y^{-} \\
= & \sum_{j=0}^{\ell-1} c_{j} y(x y)^{j} \otimes x(y x)^{\ell-1-j}+\sum_{j=0}^{\ell} \varepsilon_{i}(y x)^{j} \otimes(y x)^{\ell-j},
\end{aligned}
$$

and we replace $\lambda$ by $\lambda-\Omega_{1}^{\prime}$.

Step 3. We consider the case where $t=2 k$. We write the homogeneous component $\lambda^{(2 k)}$ in the form (2.31). Substituting this in (2.39), we obtain the equation

$$
\sum_{i=0}^{k} \alpha_{i}+\sum_{i=0}^{k-1} b_{i}+\sum_{i=1}^{k-1} \varepsilon_{i}+\sum_{i=0}^{k-1} c_{i}=0 .
$$

We repeat the arguments of cases a)-d) at Step 2; this allows us to assume that in the initial $\lambda$ the coefficients $\beta_{i}, \gamma_{i}, f_{i}$, and $a_{i}$ are zero for all corresponding values of the 
indices. Finally, we consider the following analogs of (2.40) and (2.41):

$$
\begin{aligned}
\Omega^{\prime \prime}:= & \sum_{j=0}^{k}\left(\sum_{i=k-j+1}^{k} \alpha_{i}+\sum_{i=k-j+1}^{k-1} b_{i}\right) x(y x)^{k-j} \otimes(x y)^{j-1} \cdot Y^{-} \\
& +\sum_{j=1}^{k}\left(\sum_{i=k-j+1}^{k} \alpha_{i}+\sum_{i=k-j}^{k-1} b_{i}\right)(x y)^{k-j} \otimes y(x y)^{j-1} \cdot X^{-} \\
= & \sum_{j=0}^{k} \alpha_{j}(x y)^{j} \otimes(x y)^{k-j}+\sum_{j=0}^{k-1} b_{i} x(y x)^{j} \otimes y(x y)^{k-j-1} \\
& -\left(\sum_{i=0}^{k} \alpha_{i}+\sum_{i=0}^{k-1} b_{i}\right) \cdot 1 \otimes(x y)^{k}, \\
\Omega_{1}^{\prime \prime}:= & \sum_{j=1}^{k-1}\left(\sum_{i=k-j}^{k-1} c_{i}+\sum_{i=k-j}^{k-1} \varepsilon_{i}\right) y(x y)^{k-1-j} \otimes(y x)^{j} \cdot X^{-} \\
& +\sum_{j=0}^{k-1}\left(\sum_{i=k-1-j}^{k-1} c_{i}+\sum_{i=k-j}^{k-1} \varepsilon_{i}\right)(y x)^{k-1-j} \otimes x(y x)^{j} \cdot Y^{-} \\
= & \sum_{j=0}^{k-1} c_{j} y(x y)^{j} \otimes x(y x)^{k-1-j}+\sum_{j=1}^{k-1} \varepsilon_{i}(y x)^{j} \otimes(y x)^{k-j} \\
& -\left(\sum_{i=0}^{k-1} c_{i}+\sum_{i=1}^{k} \varepsilon_{i}\right) \cdot 1 \otimes(x y)^{k} .
\end{aligned}
$$

Replacing $\lambda$ by $\lambda-\Omega^{\prime \prime}-\Omega_{1}^{\prime \prime}$ and using (2.42), we may assume that all coefficients $\alpha_{i}, b_{i}, \varepsilon_{i}, c_{i}$ are zero for the corresponding values of the indices.

Step 4. Assume that $t>2 k$. Since $\Theta$ is a homogeneous element of degree $2 k$, we have $\lambda^{(t)} \cdot \Theta=0$. On the other hand, for any homogeneous element $\zeta \in \Lambda$ of degree greater than $2 k$, we have $\mu(\zeta)=0$, whence, $\lambda^{(t)} \in \operatorname{Im} d_{0}^{Q}$.

Lemma 2.8. $\operatorname{Ker} \tau^{*}=\operatorname{Im} \sigma^{*}+\operatorname{Im}\left(Y^{-}\right)^{*}$.

Proof. It remains to prove the inclusion

$$
\operatorname{Ker} \tau^{*} \subset \operatorname{Im} \sigma^{*}+\operatorname{Im}\left(Y^{-}\right)^{*}
$$

Assume that $\lambda \in \operatorname{Ker} \tau^{*}$. We consider a nonzero homogeneous component $\lambda^{(t)}$ of the element $\lambda$ with the smallest possible $t$. Clearly, $\lambda^{(0)}=0$.

Step 1. Consider the case where $t=2 \ell+1$ with $0 \leq \ell<k-1$. We write the homogeneous component $\lambda^{(t)}$ in the form (2.23) (with $\alpha_{i}, \ldots, f_{i} \in K$ ). We present detailed arguments under the additional assumption that $\ell>0$. The proof in the case where $\ell=0$ is obtained by minor modifications.

Substitution of (2.23) in the equation

$$
\lambda \cdot \tau=0
$$


gives the following relations:

$$
\begin{aligned}
\alpha_{i}+b_{i-1} & =0 & & \text { for } 1 \leq i \leq \ell, \\
\varepsilon_{i}+c_{i} & =0 & & \text { for } 1 \leq i \leq \ell, \\
\gamma_{i-1}-\gamma_{i} & =0 & & \text { for } 2 \leq i \leq \ell, \\
a_{i-1}-a_{i} & =0 & & \text { for } 1 \leq i \leq \ell, \\
\alpha_{0}-\gamma_{1}=\beta_{0}+c_{0} & =0, & & \\
\alpha_{0}=a_{0}=a_{\ell}=\gamma_{\ell} & =0 . & &
\end{aligned}
$$

Relations (2.46) (and (2.49)) imply that

$$
\gamma_{\ell}=\gamma_{\ell-1}=\cdots=\gamma_{2}=\gamma_{1}=0 .
$$

Furthermore, relations (2.47) yield

$$
a_{\ell}=a_{\ell-1}=\cdots=a_{1}=a_{0}=0 .
$$

Now we successively put additional restrictions on $\lambda$.

a) We may assume that $\beta_{0}=c_{0}=0$. For this, we can replace $\lambda$ by $\lambda+\beta_{0} 1 \otimes(x y)^{\ell} \cdot Y^{-}$.

b) We may assume that $\alpha_{i}=0$ for $1 \leq i \leq \ell$, and $b_{i}=0$ for $0 \leq i \leq \ell-1$. For this, we can replace $\lambda$ by

$$
\lambda-\left(\sum_{i=1}^{\ell} \alpha_{i} x(y x)^{i-1} \otimes x(y x)^{\ell-i}\right) \cdot Y^{-} .
$$

c) We may assume that $\varepsilon_{i}=c_{i}=0$ for $1 \leq i \leq \ell$. For this, we can replace $\lambda$ by

$$
\lambda+\left(\sum_{i=1}^{\ell} \varepsilon_{i}(y x)^{i} \otimes(x y)^{\ell-i}\right) \cdot Y^{-} .
$$

d) We may assume that $\beta_{i}=0$ for $1 \leq i \leq \ell$. For this, we can replace $\lambda$ by

$$
\lambda+\left(\sum_{i=1}^{\ell} \beta_{i}(x y)^{i} \otimes(x y)^{\ell-i}\right) \cdot Y^{-} .
$$

e) Finally, we may assume that $f_{i}=0$ for $0 \leq i \leq \ell-1$. For this, we can replace $\lambda$ by

$$
\lambda-\left(\sum_{i=0}^{\ell-1} f_{i}(y x)^{i} \otimes(y x)^{\ell-i}\right) \cdot Y^{-} .
$$

It is clear that if the initial $\lambda$ satisfies the additional assumptions of cases a)-e), then $\lambda^{(t)}=0$.

Step 2. Now we consider the case where $t=2 \ell$ with $1 \leq \ell \leq k-1$. We write the homogeneous component $\lambda^{(t)}$ in the form (2.14). Then equation (2.43) implies the following relations:

$$
\begin{aligned}
& \alpha_{i}+b_{i-1}=0 \quad \text { for } 1 \leq i \leq \ell, \\
& \varepsilon_{i}+c_{i}=0 \quad \text { for } 1 \leq i \leq \ell-1, \\
& \gamma_{i-1}-\gamma_{i}=0 \quad \text { for } 2 \leq i \leq \ell \text {, } \\
& a_{i-1}-a_{i}=0 \quad \text { for } 1 \leq i \leq \ell-1 \text {, } \\
& \beta_{0}+c_{0}=0, \quad \alpha_{0}=\gamma_{1}=\gamma_{\ell}=a_{0}=a_{\ell-1}=0 .
\end{aligned}
$$

Slightly modifying the arguments used at Step 1, we can replace the initial $\lambda$ by an element for which all homogeneous components of degree not greater than $t$ are zero. The details of these arguments are left to the reader. 
It should be noted that, in the corresponding replacements of elements at Steps 1 and 2 , we used only elements in $\operatorname{Im} Y^{-}$.

Step 3. Put $t=2 k-1$. We consider the representation of $\lambda^{(2 k-1)}$ in the form (2.23) with $\ell=k-1$. For the coefficients in (2.23), we obtain relations (2.44)-(2.48) (with $\ell:=k-1)$, and also the relations

$$
\alpha_{0}-a_{0}=\gamma_{k-1}-a_{k-1}=a_{0}-\gamma_{k-1}=0 .
$$

Applying the arguments of Step 1, we can additionally assume that in (2.23) the following coefficients are zero: $\beta_{0}, c_{0}, \alpha_{i}$ for $1 \leq i \leq k-1, b_{i}$ for $0 \leq i \leq k-2, \varepsilon_{i}$ and $c_{i}$ for $1 \leq i \leq k-1$, and $\beta_{i}$ for $1 \leq i \leq k-1$. Furthermore, we have

$$
a:=\alpha_{0}=a_{0}=a_{1}=\cdots=a_{k-1}=\gamma_{1}=\gamma_{2}=\cdots=\gamma_{k-1} .
$$

It follows that $\lambda^{(2 k-1)}=a \cdot \sigma$.

Step 4. Put $t=2 k$. We write $\lambda^{(2 k)}$ in the form (2.31). Then equation (2.43) implies that

$$
\begin{aligned}
& \alpha_{i}+b_{i-1}=0 \quad \text { for } 1 \leq i \leq k-1 \text {, } \\
& \varepsilon_{i}+c_{i}=0 \quad \text { for } 1 \leq i \leq k-1 \text {, } \\
& \gamma_{i-1}-\gamma_{i}=0 \quad \text { for } 2 \leq i \leq k-1 \text {, } \\
& a_{i-1}-a_{i}=0 \quad \text { for } 1 \leq i \leq k-1 \text {, } \\
& \alpha_{0}-\gamma_{1}+c_{0}=0, \quad \gamma_{k-1}-\alpha_{k}-b_{k-1}=0 .
\end{aligned}
$$

As at Step 2, we may additionally assume that the following coefficients are zero: $\alpha_{i}$ for $1 \leq i \leq k-1, b_{i}$ for $0 \leq i \leq k-2, \varepsilon_{i}$ and $c_{i}$ for $1 \leq i \leq k-1, \beta_{i}$ for $1 \leq i \leq k-1$, and $f_{i}$ for $0 \leq i \leq k-1$ (the details are left to the reader).

Moreover, we may assume that $\alpha_{0}=b_{k-1}=0$. Indeed, for this, we can replace $\lambda$ by an element of the form

$$
\lambda+\left(\alpha_{0} 1 \otimes x(y x)^{k-1}+b_{k-1} x(y x)^{k-1} \otimes 1\right) \cdot Y^{-} .
$$

Finally, relations (2.52), (2.53), and (2.54) imply that

$$
\begin{aligned}
& \gamma:=\gamma_{1}=\gamma_{2}=\cdots=\gamma_{k-1}=\alpha_{k}=c_{0}, \\
& a:=a_{0}=a_{1}=\cdots=a_{k-1} .
\end{aligned}
$$

It remains to replace $\lambda$ by the element

$$
\lambda-(\gamma y \otimes 1+a x \otimes 1) \cdot \sigma .
$$

We notice that some homogeneous component of $\lambda$ of degree greater than $2 k$ is changed under this replacement.

Step 5. Now we consider the case where $t=2 \ell+1$ with $k \leq \ell \leq 2 k-1$. Put $\bar{\ell}:=l-k$. We write $\lambda^{(t)}$ in the form (2.32). We give detailed arguments for $\bar{\ell}<k-1$. If $\bar{\ell}=k-1$ (i.e., $\ell=2 k-1)$, then only minor modifications are needed. Substituting (2.32) in (2.43), we obtain

$$
\begin{aligned}
\alpha_{i}+b_{i-1} & =0 & & \text { for } \bar{\ell}+2 \leq i \leq k-1, \\
\varepsilon_{i}+c_{i} & =0 & & \text { for } \bar{\ell}+1 \leq i \leq k-1, \\
\gamma_{i-1}-\gamma_{i} & =0 & & \text { for } \bar{\ell}+2 \leq i \leq k-1, \\
a_{i-1}-a_{i} & =0 & & \text { for } \bar{\ell}+2 \leq i \leq k-1, \\
\alpha_{\bar{\ell}}+a_{\bar{\ell}}-a_{\bar{\ell}+1} & =0, & & \\
\gamma_{k-1}-b_{k-1}-\alpha_{k} & =0 . & &
\end{aligned}
$$


Again, we shall put additional restrictions on $\lambda$.

a) We may assume that $\alpha_{i}=0$ for $\bar{\ell}+2 \leq i \leq k-1$ and $b_{i}=0$ for $\bar{\ell}+1 \leq i \leq k-2$. For this, we can replace $\lambda$ by

$$
\lambda-\left(\sum_{i=\bar{\ell}+2}^{k-1} \alpha_{i} x(y x)^{i-1} \otimes x(y x)^{\ell-i}\right) \cdot Y^{-} .
$$

b) We may assume that $\varepsilon_{i}=c_{i}=0$ for $\bar{\ell}+1 \leq i \leq k-1$. For this, we can replace $\lambda$ by

$$
\lambda+\left(\sum_{i=\bar{\ell}+1}^{k-1} \varepsilon_{i}(y x)^{i} \otimes(x y)^{\ell-i}\right) \cdot Y^{-} .
$$

c) We may assume that $\beta_{i}=0$ for $\bar{\ell}+1 \leq i \leq k$. For this, we can replace $\lambda$ by

$$
\lambda+\left(\sum_{i=\bar{\ell}+1}^{k} \beta_{i}(x y)^{i} \otimes(x y)^{\ell-i}\right) \cdot Y^{-} .
$$

d) We may assume that $f_{i}=0$ for $\bar{\ell}+1 \leq i \leq k-1$. For this, we can replace $\lambda$ by

$$
\lambda-\left(\sum_{i=\bar{\ell}+1}^{k-1} f_{i}(y x)^{i} \otimes(y x)^{\ell-i}\right) \cdot Y^{-} .
$$

e) Finally, we may assume that $\alpha_{\bar{\ell}+1}=c_{\bar{\ell}}=b_{k-1}=0$. For this, we can replace $\lambda$ by

$$
\lambda-\left(\alpha_{\bar{\ell}+1} x(y x)^{\bar{\ell}} \otimes x(y x)^{k-1}+c_{\bar{\ell}}(y x)^{\bar{\ell}} \otimes(x y)^{k}-b_{k-1} x(y x)^{k-1} \otimes x(y x)^{\bar{\ell}}\right) \cdot Y^{-} .
$$

Relations (2.57) and (2.60) imply that

$$
\gamma:=\gamma_{\bar{\ell}+1}=\gamma_{\bar{\ell}+2}=\cdots=\gamma_{k-2}=\gamma_{k-1}=\alpha_{k} .
$$

Relations (2.58) and (2.59) imply that

$$
a:=a_{\bar{\ell}}=a_{\bar{\ell}+1}=\cdots=a_{k-2}=a_{k-1} .
$$

It remains to replace $\lambda$ by

$$
\lambda-\left(\gamma \cdot(y x)^{\bar{\ell}+1} \otimes 1+a \cdot 1 \otimes(x y)^{\bar{\ell}+1}\right) \cdot \sigma .
$$

Step 6. Finally, we consider the case where $t=2 \ell$ with $k<\ell \leq 2 k$.

Put $\bar{\ell}:=l-k$. We write $\lambda^{(t)}$ in the form (2.38). For $\bar{\ell}<k-1$, equation (2.43) implies the following relations:

$$
\begin{aligned}
\alpha_{i}+b_{i-1} & =0 & & \text { for } \bar{\ell}+1 \leq i \leq k-1, \\
\varepsilon_{i}+c_{i} & =0 & & \text { for } \bar{\ell}+1 \leq i \leq k-1, \\
\gamma_{i-1}-\gamma_{i} & =0 & & \text { for } \bar{\ell}+2 \leq i \leq k-1, \\
a_{i-1}-a_{i} & =0 & & \text { for } \bar{\ell}+1 \leq i \leq k-1, \\
c_{\ell}+\gamma_{\ell}-\gamma_{\ell+1} & =0, & & \\
\gamma_{k-1}-b_{k-1}-\alpha_{k} & =0 . & &
\end{aligned}
$$

Then, as in the preceding cases, we modify the initial element $\lambda$ subsequently to obtain a new element in which all homogeneous components with degree not greater than $t$ are zero.

Next, for $\bar{\ell}=k-1$, equation (2.43) implies the sole relation

$$
\alpha_{k}-\gamma_{k-1}+b_{k-1}-c_{k-1}=0 .
$$


As above, we can put successively additional restrictions on $\lambda$, which allows us to assume that $\alpha_{k-1}=0, f_{k-1}=0, \beta_{k}=0$. Furthermore, replacing $\lambda$ by

$$
\lambda-\left(c_{k-1}(y x)^{k-1} \otimes x(y x)^{k-1}-b_{k-1} x(y x)^{k-1} \otimes(x y)^{k-2}\right) \cdot Y^{-},
$$

we may also assume that $c_{k-1}=b_{k-1}=0$. Then $\alpha_{k}=\gamma_{k-1}$. It remains to replace $\lambda$ by

$$
\lambda-\left(a_{k-1} x(y x)^{k-1} \otimes 1+\gamma_{k-1}(y x)^{k-1} \otimes y\right) \cdot \sigma .
$$

Finally, for $\bar{\ell}=k$ we have $\lambda^{(4 k)} \cdot \tau=0$. It is clear that $\lambda^{(4 k)} \in \operatorname{Im} \sigma$.

Completion of the proof of Theorem 2.1. We consider the spectral sequence of the bicomplex $\mathcal{B}_{\bullet \bullet}$ in (2.10),

$$
\mathrm{H}_{p}^{v} \mathrm{H}_{q}^{h}\left(\mathcal{B}_{\bullet \bullet}\right) \quad \Rightarrow \quad \mathrm{H}_{p+q}\left(\operatorname{Tot}\left(\mathcal{B}_{\bullet \bullet}\right)\right),
$$

and prove that the second page of this spectral sequence degenerates.

By Lemma 2.4 every column with number $i>0$ on the first page of this spectral sequence contains two nonzero terms, namely,

$$
\operatorname{Coker} \tau \stackrel{\bar{\Phi}}{\longrightarrow} \operatorname{Ker}\left(Y^{-}, X^{-}\right) / \operatorname{Im} \Upsilon
$$

if $i$ is odd, and

$$
\operatorname{Coker}\left(Y^{-}, X^{-}\right) \stackrel{\bar{\sigma}}{\longrightarrow} \operatorname{Ker} \tau / \operatorname{Im} Y^{-}
$$

if $i$ is even. Here, $\bar{\Phi}$ and $\bar{\sigma}$ denote the homomorphisms induced by the homomorphisms $\Phi$ and $\sigma$, respectively. We shall prove that these homomorphisms are in fact isomorphisms.

a) $\bar{\Phi}$ is a monomorphism. Indeed, if $\Phi(\lambda) \in \operatorname{Im} \Upsilon$ with $\lambda \in \Lambda$, then

$$
\begin{aligned}
& \lambda \cdot \varphi_{1}=\lambda^{\prime} \cdot Y^{+}, \\
& \lambda \cdot \varphi_{2}=0
\end{aligned}
$$

i.e., $\left(-\lambda^{\prime}, \lambda\right) \in \operatorname{Ker} d_{1}^{Q}$. By Lemma 2.6, we have $\lambda \in \operatorname{Im} \tau$, which proves the claim.

b) $\bar{\Phi}$ is an epimorphism. Let $\left(\lambda_{1}, \lambda_{2}\right) \in \operatorname{Ker}\left(Y^{-}, X^{-}\right)$. By Lemma 2.5, there exist $q_{1}, q_{2} \in \Lambda$ such that $\left(\lambda_{1}, \lambda_{2}\right)=d_{1}^{Q}\left(q_{1}, q_{2}\right)$; i.e., we have

$$
\begin{aligned}
& \lambda_{1}=q_{1} Y^{+}+q_{2} \varphi_{1}, \\
& \lambda_{2}=q_{2} \varphi_{2} .
\end{aligned}
$$

Consequently, $\left(\lambda_{1}, \lambda_{2}\right)-\Phi\left(q_{2}\right) \in \operatorname{Im} \Upsilon$.

c) $\bar{\sigma}$ is a monomorphism. Assume that $\sigma^{*}(\lambda) \in \operatorname{Im}\left(Y^{-}\right)^{*}=\operatorname{Ker}\left(Y^{+}\right)^{*}$; i.e., $\lambda \in$ $\operatorname{Ker}\left(\sigma Y^{+}\right)^{*}$. By Lemma 2.7. $\lambda \in \operatorname{Im} d_{0}^{Q}=\operatorname{Im}\left(Y^{-}, X^{-}\right)$.

d) $\bar{\sigma}$ is an epimorphism. Let $\lambda \in \operatorname{Ker} \tau$. By Lemma 2.8, there exist $q_{1}, q_{2} \in \Lambda$ such that $\lambda=q_{1} \sigma+q_{2} Y^{-}$. Consequently, $\bar{\sigma}\left(q_{1}+\operatorname{Im}\left(Y^{-}, X^{-}\right)\right)=\lambda+\operatorname{Im} Y^{-}$.

Finally, in the 0th column of the first page of the spectral sequence, there is a unique nonzero term $\operatorname{Coker}\left(Y^{-}, X^{-}\right) \simeq R$. Consequently, we have proved that $\mu: Q_{\bullet} \stackrel{\mu}{\longrightarrow} R$ is a $\Lambda$-projective resolution of the module $R$. Its minimality follows immediately from the construction.

Corollary 2.9. Let $l_{n}$ denote the rank of the free $\Lambda$-module $Q_{n}$. Then

$$
l_{n}= \begin{cases}1+2\left[\frac{n}{4}\right] & \text { if } n \equiv 0(\bmod 4), \\ 2+2\left[\frac{n}{4}\right] & \text { in other cases. }\end{cases}
$$


Remark 2.10. Let $Q . \stackrel{\mu}{\longrightarrow} R$ be the minimal $\Lambda$-projective resolution of the algebra $R$, as described in Theorem 2.1. In the sequel, in the decomposition of the $m$ th member $Q_{m}=\bigoplus_{i+j=m} B_{i j}$ of this resolution, we always arrange the direct summands with respect to the increase of the second index $j$. The corresponding decomposition of the module $Q_{m}$ is said to be standard.

We consider the bicomplex $\mathcal{A}_{\text {.• }}$ that consists of the first two rows of the bicomplex $\mathcal{B}_{\bullet \bullet}$ with numbers 0 and 1 (the other rows in $\mathcal{A}_{\bullet \bullet}$ are zero). Set $X_{\bullet}=\operatorname{Tot}\left(\mathcal{A}_{\bullet \bullet}\right)$.

Proposition 2.11. There is a short exact sequence of complexes

$$
0 \rightarrow X_{\bullet} \stackrel{i}{\longrightarrow} Q \bullet \stackrel{\pi}{\longrightarrow} Q \bullet[-4] \rightarrow 0
$$

that splits in each degree.

Proof. Let $i_{n}: X_{n} \rightarrow Q_{n}$ be the inclusion of the direct summand, and let $\pi_{n}: Q_{n} \rightarrow Q_{n-4}$ be the projection onto the corresponding direct summand $(n \geq 4)$. Then the statement follows immediately from the construction of the bicomplex $\mathcal{B} .$. .

\section{§3. Additive structure of the cohomology algebra}

In the sequel, we assume that $\operatorname{char} K=2$. Put $R=R_{k}(k \geq 2)$. Let $Q \bullet \stackrel{\mu}{\longrightarrow} R$ be the minimal $\Lambda$-projective resolution of the algebra $R$, described in 92 To calculate the cohomology $\mathrm{HH}^{n}(R)$, we use the complex

$$
\left(\operatorname{Hom}_{\Lambda}\left(Q_{n}, R\right), \delta^{n}=\operatorname{Hom}_{\Lambda}\left(d_{n}^{Q}, R\right)\right)_{n \geq 0} .
$$

For any $n \geq 0$, the module $Q_{n}$ is a free $\Lambda$-module of rank $l_{n}$ (see Corollary [2.9); hence, any element $f$ in $\operatorname{Hom}_{\Lambda}\left(Q_{n}, R\right)$ is identified with the collection of its values $f\left(e^{(i)}\right) \in R$ at the elements of the canonical basis of the module $Q_{n}$ (that is, the $i$ th coordinate of $e^{(i)}$ is equal to $1 \otimes 1$, and the others are zero).

We observe that if $f=w^{*}: \Lambda \rightarrow \Lambda$ is a homomorphism of multiplication on the right by $w \in \Lambda$, then, in accordance with the identification mentioned above, the induced homomorphism of Abelian groups

$$
\operatorname{Hom}_{\Lambda}(f, R): \operatorname{Hom}(\Lambda, R) \simeq R \rightarrow \operatorname{Hom}(\Lambda, R) \simeq R
$$

acts as follows: $r \in R$ is taken to $w * r$ (here, $*$ corresponds to the $\Lambda$-module structure on $R$ ).

We simplify the notation in (2.2), (2.3), (2.6), (2.7), denoting $Y^{ \pm}, X^{-}$, and $\rho^{ \pm}$by $Y, X$, and $\rho$, respectively. Then the differential

$$
\delta^{0}: \operatorname{Hom}_{\Lambda}\left(Q_{0}, R\right) \simeq R \rightarrow \operatorname{Hom}_{\Lambda}\left(Q_{1}, R\right) \simeq R^{2}
$$

can be described by the following formula:

$$
\delta^{0}(r)=(Y * r, X * r)=(y r-r y, x r-r x)
$$

for any $r \in R$.

Proposition 3.1. 1) The following set is a $K$-basis of the vector space $\mathrm{HH}^{0}(R)$ :

$$
\left\{(x y)^{i}+(y x)^{i} \mid 1 \leq i \leq k-1\right\} \cup\left\{1, x(y x)^{k-1}, y(x y)^{k-1},(x y)^{k}\right\} .
$$

2) The set formed by the elements

$$
\begin{gathered}
\left((x y)^{i}+(y x)^{i}, 0\right) \quad \text { for } 1 \leq i \leq k-1, \\
\left(0,(x y)^{i}+(y x)^{i}\right) \quad \text { for } 1 \leq i \leq k-1, \quad\left(y(x y)^{i}, x(y x)^{i}\right) \quad \text { for } 1 \leq i \leq k-1
\end{gathered}
$$

is a $K$-basis for the vector space $\operatorname{Im} \delta^{0}$. 
Proof. The first statement follows from [4, III.14]. To prove the second statement, it suffices to calculate $\delta^{0}(r)$ for $r$ running through the standard basis of the algebra $R$ and then to pick a basis in the resulting collection of values of the differential $\delta^{0}$.

Corollary 3.2. $\operatorname{dim}_{K} \mathrm{HH}^{0}(R)=k+3$, and $\operatorname{dim}_{K} B^{1}(R)=3 k-3$.

Remark 3.3. In the sequel, we use a brief notation for several elements in (3.2):

$$
p_{1}:=x y+y x, \quad p_{2}:=x(y x)^{k-1}, \quad p_{3}:=y(x y)^{k-1}, \quad p_{4}:=(x y)^{k} .
$$

Observe that $p_{1}^{i}=(x y)^{i}+(y x)^{i}(1 \leq i \leq k-1), p_{1}^{k}=2(x y)^{k}=0$.

The differential

$$
\delta^{1}: \operatorname{Hom}_{\Lambda}\left(Q_{1}, R\right) \rightarrow \operatorname{Hom}_{\Lambda}\left(Q_{2}, R\right)
$$

can be described as follows: for any $r_{1}, r_{2} \in R$, we have

$$
\delta^{1}\left(r_{1}, r_{2}\right)=\left(Y * r_{1}, \varphi_{1} * r_{1}+\varphi_{2} * r_{2}\right) .
$$

Now, assume that $\left(r_{1}, r_{2}\right) \in \operatorname{Ker} \delta^{1}$, i.e.,

$$
\begin{aligned}
Y * r_{1}=y r_{1}+r_{1} y & =0, \\
\varphi_{1} * r_{1}+\varphi_{2} * r_{2}= & \sum_{i=0}^{k-1}(y x)^{i} \cdot r_{1} \cdot(x y)^{k-1-i} \\
& +x r_{2}+r_{2} x+\sum_{i=0}^{k-2}(y x)^{i} y \cdot r_{2} \cdot y(x y)^{k-2-i}=0 .
\end{aligned}
$$

We write the components of this 1-cocycle in the form

$$
r_{1}=\sum_{w \in \mathcal{B}_{R}} \lambda_{w} w, \quad r_{2}=\sum_{w \in \mathcal{B}_{R}} \mu_{w} w
$$

$\left(\lambda_{w}, \mu_{w} \in K\right)$. The first equation in (3.6) yields the following system of equations for the coordinates of the expansions in (3.7):

$$
\begin{aligned}
\lambda_{(x y)^{i}}+\lambda_{(y x)^{i}}=0 & \text { for } \quad 1 \leq i \leq k-1, \\
\lambda_{x(y x)^{i}}=0 & \text { for } \quad 0 \leq i \leq k-2 .
\end{aligned}
$$

Next, the second equation in (3.6) is equivalent to the system

$$
\begin{aligned}
\mu_{(x y)^{i}}+\mu_{(y x)^{i}} & =0 \quad \text { for } 1 \leq i \leq k-1, \\
\mu_{y(x y)^{i}} & =0 \quad \text { for } 0 \leq i \leq k-3, \\
\lambda_{1}+\mu_{y(x y)^{k-2}} & =0, \\
k \cdot \lambda_{y}+(k+1) \cdot \mu_{x} & =0 .
\end{aligned}
$$

Now, analyzing relations (3.8) and (3.9), we obtain the following statement. 
Proposition 3.4. a) Assume that $k$ is even. Then the elements

$$
\begin{array}{lr}
\left((x y)^{i}+(y x)^{i}, 0\right) & \text { for } 1 \leq i \leq k-1, \\
\left(0,(x y)^{i}+(y x)^{i}\right) & \text { for } 1 \leq i \leq k-1, \\
\left(y(x y)^{i}, 0\right) & \text { for } 0 \leq i \leq k-1, \\
\left(0, x(y x)^{i}\right) & \text { for } 1 \leq i \leq k-1, \\
\left(1, y(x y)^{k-2}\right),\left(x(y x)^{k-1}, 0\right),\left((x y)^{k}, 0\right), & \\
(0,1),\left(0, y(x y)^{k-1}\right),\left(0,(x y)^{k}\right) &
\end{array}
$$

form a $K$-basis of the vector space $\operatorname{Ker} \delta^{1}$.

b) Assume that $k$ is odd. To obtain a basis of the vector space $\operatorname{Ker} \delta^{1}$, it suffices to replace the element $(y, 0)$ in the set described above by $(0, x)$.

c) For any $k \geq 2$, the elements

$$
\begin{array}{ll}
\left((x y)^{i}+(y x)^{i}, 0\right) & \text { for } 1 \leq i \leq k-1, \\
\left(y(x y)^{i}, 0\right) & \text { for } 2 \leq i \leq k-1, \\
\left(0,(x y)^{i}+(y x)^{i}\right) & \text { for } 1 \leq i \leq k-1, \\
\left(0, x(y x)^{i}\right) & \text { for } 1 \leq i \leq k-1, \\
\left(y x y,(x y)^{k}\right),\left(0, y(x y)^{k-1}\right) &
\end{array}
$$

form a $K$-basis of the vector space $\operatorname{Im} \delta^{1}$.

Corollary 3.5. $\operatorname{dim}_{K} \operatorname{Ker} \delta^{1}=4 k+3$, and $\operatorname{dim}_{K} \operatorname{Im} \delta^{1}=4 k-3$.

Proposition 3.6. a) Assume that $k$ is even. Then the cohomology classes of the elements listed in (3.12), (3.14), and (3.15) form a $K$-basis of the vector space $\mathrm{HH}^{1}(R)$.

b) Assume that $k$ is odd. To obtain a basis of the vector space $\mathrm{HH}^{1}(R)$, it suffices to replace the cohomology class of the element $(y, 0)$ in the set indicated in a) by the cohomology class of the element $(0, x)$.

Proof. This follows from Proposition 3.4 and Proposition 3.1 part (2).

Corollary 3.7. $\operatorname{dim}_{K} \mathrm{HH}^{1}(R)=k+6$.

Under the above assumptions, the differential $\delta^{2}$ is described by the following rule: for any $r_{1}, r_{2} \in R$, we have

$$
\delta^{2}\left(r_{1}, r_{2}\right)=\left(Y * r_{1}, \rho * r_{1}+\tau * r_{2}\right) .
$$

Assume that $\left(r_{1}, r_{2}\right) \in \operatorname{Ker} \delta^{2}$, i.e.,

$$
Y * r_{1}=0, \quad \rho * r_{1}+\tau * r_{2}=0 .
$$

We represent the components of this 2-cocycle in the form (3.7). The first equation in (3.21) is equivalent to (3.8), and the second is equivalent to the relation

$$
\mu_{(x y)^{i}}+\mu_{(y x)^{i}}=0 \text { for } 1 \leq i \leq k-2
$$

(it should be noted that $\rho * r=0$ for any $r \in R$ ). 
Arguing as above, it is easy to prove the following statements.

Proposition 3.8. 1) The elements

$$
\begin{array}{lr}
\left((x y)^{i}+(y x)^{i}, 0\right) & \text { for } 1 \leq i \leq k-1 \\
\left(0,(x y)^{i}+(y x)^{i}\right) & \text { for } 1 \leq i \leq k-2, \\
\left(y(x y)^{i}, 0\right) & \text { for } 0 \leq i \leq k-1 \\
\left(0, x(y x)^{i}\right) & \text { for } 0 \leq i \leq k-1, \\
\left(0, y(x y)^{i}\right) & \text { for } 0 \leq i \leq k-1, \\
(1,0),\left(x(y x)^{k-1}, 0\right),\left((x y)^{k}, 0\right), & \\
(0,1),\left(0,(x y)^{k-1}\right), & \\
\left(0,(y x)^{k-1}\right),\left(0,(x y)^{k}\right) &
\end{array}
$$

form a K-basis of the vector space $\operatorname{Ker} \delta^{2}$.

2) The elements

$$
\begin{array}{ll}
\left((x y)^{i}+(y x)^{i}, 0\right) & \text { for } 1 \leq i \leq k-1, \\
\left(0,(x y)^{i}+(y x)^{i}\right) & \text { for } 2 \leq i \leq k-1, \\
\left(y(x y)^{i}, 0\right) & \text { for } 1 \leq i \leq k-1
\end{array}
$$

form a K-basis of the vector space $\operatorname{Im} \delta^{2}$.

Corollary 3.9. $\operatorname{dim}_{K} \operatorname{Ker} \delta^{2}=5 k+4$, and $\operatorname{dim}_{K} \operatorname{Im} \delta^{2}=3 k-4$.

Proposition 3.10. A $K$-basis of the vector space $\mathrm{HH}^{2}(R)$ can be formed by the cohomology classes of the elements listed in (3.27) and (3.28) and the cohomology classes of the elements

$$
\begin{array}{ll}
\left(0, y(x y)^{i}\right) & \text { for } 0 \leq i \leq k-2, \\
(y, 0),(0, x),(y x y, 0) &
\end{array}
$$

Corollary 3.11. $\operatorname{dim}_{K} \mathrm{HH}^{2}(R)=k+7$.

The differential

$$
\delta^{3}: \operatorname{Hom}_{\Lambda}\left(Q_{3}, R\right) \rightarrow \operatorname{Hom}_{\Lambda}\left(Q_{4}, R\right)
$$

can be described as follows: for any $r_{1}, r_{2} \in R$,

$$
\delta^{3}\left(r_{0}, r_{1}\right)=\left(Y * r_{1}, \rho * r_{1}+Y * r_{2}, \sigma * r_{2}\right) .
$$

The condition $\left(r_{1}, r_{2}\right) \in \operatorname{Ker} \delta^{3}$ is equivalent to the relations

$$
Y * r_{1}=0, Y * r_{2}=0 .
$$

Here, we use the fact that $\rho * r=0=\sigma * r$ for any $r \in R$. Representing the components $r_{1}, r_{2}$ of this cocycle in the form (3.7), we obtain relations connecting the coefficients $\lambda_{w}, \mu_{w}\left(w \in \mathcal{B}_{R}\right)$ in the corresponding decompositions. Now, we easily establish the following statements. 
Proposition 3.12. 1) The elements

$$
\begin{array}{lr}
\left((x y)^{i}+(y x)^{i}, 0\right) & \text { for } 1 \leq i \leq k-1, \\
\left(0,(x y)^{i}+(y x)^{i}\right) & \text { for } 1 \leq i \leq k-1, \\
\left(y(x y)^{i}, 0\right) & \text { for } 0 \leq i \leq k-1, \\
\left(0, y(x y)^{i}\right) & \text { for } 0 \leq i \leq k-1, \\
(1,0),\left(x(y x)^{k-1}, 0\right),\left((x y)^{k}, 0\right), & \\
(0,1),\left(0, x(y x)^{k-1}\right),\left(0,(x y)^{k}\right) &
\end{array}
$$

form a $K$-basis of the vector space $\operatorname{Ker} \delta^{3}$.

2) The elements

$$
\begin{array}{ll}
\left((x y)^{i}+(y x)^{i}, 0,0\right) & \text { for } 1 \leq i \leq k-1, \\
\left(0,(x y)^{i}+(y x)^{i}, 0\right) & \text { for } 1 \leq i \leq k-1, \\
\left(y(x y)^{i}, 0,0\right) & \text { for } 1 \leq i \leq k-1, \\
\left(0, y(x y)^{i}, 0\right) & \text { for } 1 \leq i \leq k-1
\end{array}
$$

form a $K$-basis of the vector space $\operatorname{Im} \delta^{3}$.

Corollary 3.13. $\operatorname{dim}_{K} \operatorname{Ker} \delta^{3}=4 k+4$, and $\operatorname{dim}_{K} \operatorname{Im} \delta^{3}=4 k-4$.

Proposition 3.14. The classes of the elements listed in (3.39), (3.40), and (3.41), and the cohomology classes of the elements

$$
(y, 0),(0, x y+y x)
$$

form a $K$-basis of the vector space $\mathrm{HH}^{3}(R)$.

Corollary 3.15. $\operatorname{dim}_{K} \mathrm{HH}^{3}(R)=k+8$.

Proposition 3.16. For any $n \geq 4$, we have

$$
\operatorname{dim}_{K} \mathrm{HH}^{n}(R)=\operatorname{dim}_{K} \mathrm{HH}^{n-4}(R)+8 .
$$

Proof. Proposition 2.11 implies that we have the following exact sequence of complexes:

$$
0 \rightarrow \operatorname{Hom}_{\Lambda}\left(Q_{\bullet}[-4], R\right) \stackrel{\pi^{*}}{\longrightarrow} \operatorname{Hom}_{\Lambda}(Q \bullet, R) \stackrel{i^{*}}{\rightarrow} \mathcal{X}^{\bullet} \rightarrow 0,
$$

where $\mathcal{X}^{\bullet}=\operatorname{Hom}_{\Lambda}\left(X_{\bullet}, R\right)$.

In its turn, this sequence leads to the long exact cohomology sequence

$$
\ldots \stackrel{\Delta^{n-1}}{\longrightarrow} \mathrm{HH}^{n-4}(R) \stackrel{\pi^{*}}{\longrightarrow} \mathrm{HH}^{n}(R) \stackrel{i^{*}}{\longrightarrow} \mathrm{H}^{n}\left(\mathcal{X} \bullet \stackrel{\Delta^{n}}{\longrightarrow} \mathrm{HH}^{n-3}(R) \stackrel{\pi^{*}}{\longrightarrow} \cdots .\right.
$$

Now, we need to compute the cohomology of the auxiliary complex $\mathcal{X}^{\bullet}$.

Lemma 3.17. For any $n \geq 4$, we have

$$
\operatorname{dim}_{K} \mathrm{H}^{n}\left(\mathcal{X}^{\bullet}\right)=8 \text {. }
$$

Proof. As usual, we identify $\mathcal{X}^{n}=\operatorname{Hom}_{\Lambda}\left(X_{n}, R\right)$, where $n \geq 1$, and $R^{2}$ (but $\mathcal{X}^{0}$ is identified with $R$ ). Let $f_{y}: R \rightarrow R$ denote the homomorphism (of Abelian groups) such that $f_{y}(r)=Y * r=y r+r y$. For $n \geq 3$, the differential $d_{\mathcal{X}}^{n}$. is described by the formula 
$d_{\mathcal{X}}^{n}\left(r_{1}, r_{2}\right)=\left(f_{y}\left(r_{1}\right), f_{y}\left(r_{2}\right)\right)$. Therefore, for $n \geq 4$ we have $\mathrm{H}^{n}\left(\mathcal{X}^{\bullet}\right) \simeq\left(\operatorname{Ker} f_{y} / \operatorname{Im} f_{y}\right)^{2}$. Arguing as in the proof of Proposition 3.4, we see that $\operatorname{Ker} f_{y} / \operatorname{Im} f_{y}$ is generated by the classes of the elements $1, y, x(y x)^{k-1},(x y)^{k}$, and (3.49) follows.

We continue the proof of Proposition 3.16. Lemma 3.17) shows that, to establish (3.47), it suffices to prove that the connecting homomorphisms $\Delta^{n}$ in the sequence (3.48) are zero if $n \geq 3$.

We consider the following diagram, which is used in the construction of the connecting homomorphism:

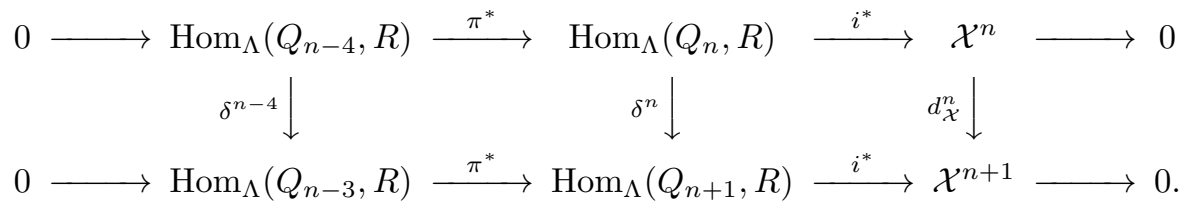

Let $f=\left(r_{1}, r_{2}\right) \in \mathcal{X}^{n}$ be an $n$-cocycle, $n \geq 3$. We denote by $\nu: Q_{n}=X_{n} \oplus Q_{n-4} \rightarrow X_{n}$ the projection onto the direct summand. Then we define $\widetilde{f}=f \circ \nu: Q_{n} \rightarrow R$; in other words, the map $\tilde{f}$ is obtained from $f$ via extension by zero to the direct summand complementary to $X_{n}$ in $Q_{n}$, i.e., $\widetilde{f}=\left(r_{1}, r_{2}, \mathrm{O}\right)$. It is clear that $i^{*}(\widetilde{f})=f$. If $n \geq 4$, then the matrix of the differential $d_{n}^{Q}$ has the following block structure:

$$
d_{n}^{Q}=\left(\begin{array}{cc|cc}
Y & \rho & 0 & \mathrm{O}_{1, l^{\prime}-3} \\
0 & Y & \rho & \mathrm{O}_{1, l^{\prime}-3} \\
\hline \mathrm{O}_{l-2,2} & & d_{n-4}^{Q}
\end{array}\right)
$$

with $l=l_{n}, l^{\prime}=l_{n+1}$ (see formula (2.68) $)$. In this case, we see that

$$
\delta^{n}(\widetilde{f})=\left(Y * r_{1}, Y * r_{2}, \mathrm{O}_{l^{\prime}-2}\right)=0,
$$

whence $\Delta^{n}(\operatorname{cl} f)=0$. If $n=3$, then (3.35) shows directly that $\delta^{3}(\widetilde{f})=0$; consequently, $\Delta^{3}(\operatorname{cl} f)=0$.

Corollary 3.18. Put $R=R_{k}$. Then

$$
\operatorname{dim}_{K} \mathrm{HH}^{n}(R)= \begin{cases}k+3+8\left[\frac{n}{4}\right] & \text { if } n \equiv 0(\bmod 4), \\ k+6+8\left[\frac{n}{4}\right] & \text { if } n \equiv 1(\bmod 4), \\ k+7+8\left[\frac{n}{4}\right] & \text { if } n \equiv 2(\bmod 4), \\ k+8+8\left[\frac{n}{4}\right] & \text { if } n \equiv 3(\bmod 4) .\end{cases}
$$

In the course of the proof of Proposition 3.16, for $n \geq 4$, we established isomorphism

$$
\mathrm{HH}^{n}(R) \simeq \mathrm{HH}^{n-4}(R) \oplus \mathrm{H}^{n}\left(\mathcal{X}^{\bullet}\right) .
$$

This isomorphism and the proof of Lemma 3.17 lead to the following two statements.

Proposition 3.19. The vector space $\mathrm{HH}^{4}(R)$ has a $K$-basis formed by the cohomology classes of the following elements:

$$
\begin{aligned}
& (1,0,0),(y, 0,0),\left(x(y x)^{k-1}, 0,0\right),\left((x y)^{k}, 0,0\right) \\
& (0,1,0),(0, y, 0),\left(0, x(y x)^{k-1}, 0\right),\left(0,(x y)^{k}, 0\right), \\
& \left(0,0,(x y)^{i}+(y x)^{i}\right) \\
& (0,0,1),\left(0,0, x(y x)^{k-1}\right),\left(0,0, y(x y)^{k-1}\right),\left(0,0,(x y)^{k}\right) .
\end{aligned}
$$


Proposition 3.20. a) If $k$ is even, then the vector space $\operatorname{HH}^{5}(R)$ has a K-basis formed by the cohomology classes of the following elements:

$$
\begin{aligned}
& \left(1, \mathrm{O}_{3}\right),\left(y, \mathrm{O}_{3}\right),\left(x(y x)^{k-1}, \mathrm{O}_{3}\right),\left((x y)^{k}, \mathrm{O}_{3}\right), \\
& \left(0,1, \mathrm{O}_{2}\right),\left(0, y, \mathrm{O}_{2}\right),\left(0, x(y x)^{k-1}, \mathrm{O}_{2}\right),\left(0,(x y)^{k}, \mathrm{O}_{2}\right), \\
& \left(\mathrm{O}_{2}, y(x y)^{i}\right) \\
& \left(\mathrm{O}_{2}, 1, y(x y)^{k-2}\right),\left(\mathrm{O}_{2}, x(y x)^{k-1}, 0\right),\left(\mathrm{O}_{2},(x y)^{k}, 0\right), \\
& \left(\mathrm{O}_{3}, 1\right),\left(\mathrm{O}_{3}, y(x y)^{k-1}\right),\left(\mathrm{O}_{3},(x y)^{k}\right) .
\end{aligned}
$$

b) If $k$ is odd, then, in order to get a basis of the vector space $\operatorname{HH}^{5}(R)$, it suffices to replace the cohomology class of the element $\left(\mathrm{O}_{2}, y, 0\right)$ in the set described above in part a) by the cohomology class of the element $\left(\mathrm{O}_{3}, x\right)$.

\section{§4. Generators and Relations}

We recall briefly an interpretation of the Yoneda product in the algebra

$$
\operatorname{HH}^{*}(R)=\bigoplus_{m \geq 0} \operatorname{Ext}_{\Lambda}^{m}(R, R),
$$

used in [3]. Let

$$
\mu: Q \bullet \rightarrow R
$$

be the minimal $\Lambda$-projective resolution described in $\S 2$. Let

$$
\operatorname{Hom}_{\Lambda}\left(Q_{\bullet}, R\right)=\left(\operatorname{Hom}_{\Lambda}\left(Q_{n}, R\right), \delta^{n}\right)
$$

be the complex (3.1). Then for cocycles $f \in \operatorname{Ker} \delta^{n}$ and $g \in \operatorname{Ker} \delta^{t}$ we have

$$
\operatorname{cl} g \cdot \operatorname{cl} f=\operatorname{cl}\left(\mu \mathrm{T}^{0}(g) \mathrm{T}^{t}(f)\right),
$$

where $T^{i}(h)$ denotes the $i$ th translate of the cocycle $h$. In the sequel, we shall describe the translates $\mathrm{T}^{i}(h)(i \geq 0)$ with the help of matrices that correspond to the standard decompositions of the modules $Q_{i}$ (see Remark 2.10).

Case 1. First, assume that $k$ is even. We consider the following homogeneous elements of $\mathrm{HH}^{*}(R)$ :

$$
\begin{array}{ll}
\text { — of degree } 0: & p_{1}, p_{2}, p_{3}, p_{4} \text { in (3.5); } \\
\text { - of degree 1: } & u_{1}:=\left(1, y(x y)^{k-2}\right), u_{2}:=(0,1), u_{3}:=(y, 0) ; \\
\text { — of degree 2: } & v_{1}:=(y, 0), v_{2}:=(0, y), v_{3}:=(0, x) ; \\
\text { — of degree } 3: & w_{1}:=(0,1), w_{2}:=(0, y) ; \\
\text { - of degree } 4: & t:=(0,0,1) .
\end{array}
$$

Proposition 4.1. Assume that $k$ is even. In the algebra $\mathrm{HH}^{*}(R)$, the elements of the set

$$
\mathcal{Y}_{1}=\left\{p_{1}, p_{2}, p_{3}, p_{4}, u_{1}, u_{2}, u_{3}, v_{1}, v_{2}, v_{3}, w_{1}, w_{2}, t\right\}
$$


satisfy the following relations:

$$
\begin{gathered}
p_{1}^{k}=p_{2}^{2}=p_{3}^{2}=p_{4}^{2}=0, \\
p_{i} p_{j}=0 \text { for } 1 \leq i<j \leq 4 ; \\
p_{1} u_{1}=p_{3} u_{2}, p_{3} u_{1}=p_{2} u_{2}, p_{1} u_{2}=0, \\
p_{3} u_{3}=p_{4} u_{3}=0, p_{4} u_{1}=p_{2} u_{3}, p_{2} u_{2}=p_{1}^{k-1} u_{3} ; \\
p_{3} v_{1}=p_{4} v_{1}=p_{1}^{k-1} v_{2}=p_{3} v_{2}=p_{4} v_{2}=0, p_{1} v_{1}=p_{2} v_{2}, \\
p_{2} v_{1}=p_{4} u_{1}^{2}, \\
p_{1} v_{3}=p_{2} v_{3}=p_{4} v_{3}=0, \\
u_{1} u_{2}=u_{2} u_{3}=u_{3}^{2}=0, p_{2} v_{2}=p_{3} v_{3}=p_{4} u_{2}^{2} ; \\
p_{3} w_{2}=p_{4} w_{2}=0, \\
u_{2}^{3}=u_{2} v_{2}=0, p_{1} w_{1}=u_{1} v_{2}, p_{3} w_{1}=u_{2} v_{1}, p_{4} w_{1}=p_{2} w_{2}, \\
u_{3} v_{3}=0, p_{3} w_{1}=u_{1} v_{3}=p_{1}^{k-2} u_{3} v_{2}, p_{1} w_{1}=u_{2} v_{3}, \\
p_{4} w_{1}=u_{3} v_{1}, p_{1} w_{2}=u_{3} v_{2}, u_{1}^{2} u_{3}=u_{1} v_{1}+p_{2} w_{1} ; \\
v_{1}^{2}=v_{1} v_{2}=0, v_{2}^{2}=p_{1}^{2} t, \\
v_{1} v_{3}=v_{2} v_{3}=v_{3}^{2}=0, \\
u_{2} w_{1}=u_{2} w_{2}=u_{3} w_{2}=0, u_{1} w_{2}=u_{3} w_{1} ; \\
v_{2} w_{1}=p_{3} u_{2} t, \\
v_{3} w_{2}=0, v_{1} w_{2}=p_{4} u_{1} t, v_{2} w_{2}=p_{1} u_{3} t, v_{3} w_{1}=p_{3} u_{1} t \\
u_{1} u_{3} w_{1}=\left\{\begin{array}{c}
v_{1} w_{1}+p_{2} u_{1} t \\
v_{1} w_{1}+\left(p_{2} u_{1}+p_{4} u_{2}\right) t \quad \text { if } k=2 ; \\
w_{2}^{2}=0, w_{1}^{2}=u_{1}^{2} t, \\
w_{1} w_{2}=u_{1} u_{3} t .
\end{array}\right.
\end{gathered}
$$

Proof. Relations (4.6), (4.7), (4.8), (4.9), (4.11), and (4.13) are verified directly. To prove the remaining relations, we need to compute the translates of suitable order for the elements in $\mathcal{Y}_{1}$ that have positive degree.

Proposition 2.11 implies the following statement.

Lemma 4.2. For any $i \geq 0$, the projection onto the direct summand $\pi_{i+4}: Q_{i+4}=$ $X_{i+4} \oplus Q_{i} \rightarrow Q_{i}$ is the ith translate $\mathrm{T}^{i}(t)$ of the cocycle $t$.

Suitable translates of the other elements in $\mathcal{Y}_{1}$ are presented in the following lemma.

Lemma 4.3. For the role of translates of the elements in $\mathcal{Y}_{1} \backslash\{t\}$ that have a positive degree, we can take the homomorphisms determined by the following matrices:

$$
\begin{aligned}
& \mathrm{T}^{0}\left(u_{1}\right)=\left(1 \otimes 1, y(x y)^{k-2} \otimes 1\right), \\
& \mathrm{T}^{1}\left(u_{1}\right)=\left(\begin{array}{cc}
1 \otimes 1 & \sum_{i=0}^{k-2} x(y x)^{i} \otimes(x y)^{k-2-i} \\
0 & \sum_{i=0}^{k-2} y(x y)^{i} \otimes(x y)^{k-2-i}+\sum_{i=0}^{k-2}(x y)^{i} \otimes y(x y)^{k-2-i}
\end{array}\right), \\
& \mathrm{T}^{2}\left(u_{1}\right)=\left(\begin{array}{cc}
1 \otimes 1 & (y x)^{3 k-5} \otimes y \\
0 & x \otimes 1+1 \otimes x+y \otimes y(x y)^{k-2}
\end{array}\right), \\
& \mathrm{T}^{3}\left(u_{1}\right)=\left(\begin{array}{ccc}
1 \otimes 1 & 0 & \star \\
0 & 1 \otimes 1 & \star
\end{array}\right)
\end{aligned}
$$


with

$$
\begin{aligned}
& \left(\mathrm{T}^{3}\left(u_{1}\right)\right)_{13}= \begin{cases}0 & \text { if } k>2, \\
x y x \otimes x y+(x y)^{2} \otimes x & \text { if } k=2,\end{cases} \\
& \left(\mathrm{T}^{3}\left(u_{1}\right)\right)_{23}=\sum_{i=0}^{k-2} x(y x)^{i} \otimes x(y x)^{k-2-i}+(y x)^{k-1} \otimes y(x y)^{k-2}+y(x y)^{k-2} \otimes(x y)^{k-1} ; \\
& \mathrm{T}^{0}\left(u_{2}\right)=(0,1 \otimes 1), \quad \mathrm{T}^{1}\left(u_{2}\right)=\left(\begin{array}{cc}
0 & \sum_{i=0}^{k-2}(y x)^{i} \otimes y(x y)^{k-2-i} \\
0 & 1 \otimes 1
\end{array}\right), \\
& \mathrm{T}^{2}\left(u_{2}\right)=\left(\begin{array}{cc}
0 & 1 \otimes(x y)^{k-1}+1 \otimes(y x)^{k-1} \\
0 & y \otimes 1+1 \otimes y
\end{array}\right) ; \\
& \mathrm{T}^{0}\left(u_{3}\right)=(y \otimes 1,0), \quad \mathrm{T}^{1}\left(u_{3}\right)=\left(\begin{array}{cc}
y \otimes 1 & \sum_{i=1}^{k-1} i(y x)^{i} \otimes(x y)^{k-1-i} \\
0 & \sum_{i=0}^{k-2}(i+1) y(x y)^{i} \otimes y(x y)^{k-2-i}
\end{array}\right) ; \\
& \mathrm{T}^{0}\left(v_{1}\right)=(y \otimes 1,0), \quad \mathrm{T}^{1}\left(v_{1}\right)=\left(\begin{array}{cc}
y \otimes 1 & \sum_{i=1}^{k-1}(y x)^{i} \otimes x(y x)^{k-1-i} \\
0 & \sum_{i=0}^{k-1} y(x y)^{i} \otimes(y x)^{k-1-i}
\end{array}\right), \\
& \mathrm{T}^{2}\left(v_{1}\right)=\left(\begin{array}{ccc}
y \otimes 1 & \rho & \star \\
0 & 1 \otimes x y+x \otimes y & \star
\end{array}\right)
\end{aligned}
$$

with

$$
\begin{aligned}
& \left(\mathrm{T}^{2}\left(v_{1}\right)\right)_{13}= \begin{cases}0 & \text { if } k>2, \\
x y x \otimes y x y+y x \otimes(x y)^{2} & \text { if } k=2,\end{cases} \\
& \left(\mathrm{T}^{2}\left(v_{1}\right)\right)_{23}=y(x y)^{k-1} \otimes(y x)^{k-1}+y(x y)^{k-1} \otimes(x y)^{k-1} ; \\
& \mathrm{T}^{0}\left(v_{2}\right)=(0, y \otimes 1), \quad \mathrm{T}^{1}\left(v_{2}\right)=\left(\begin{array}{cc}
0 & y x \otimes 1+x \otimes y \\
0 & y \otimes y
\end{array}\right), \\
& \mathrm{T}^{2}\left(v_{2}\right)=\left(\begin{array}{ccc}
0 & 0 & y(x y)^{k-1} \otimes(x y)^{k-1} \\
0 & 0 & X \cdot(x \otimes y+y \otimes x)
\end{array}\right) ; \\
& \mathrm{T}^{0}\left(v_{3}\right)=(0,1 \otimes x), \quad \mathrm{T}^{1}\left(v_{3}\right)=\left(\begin{array}{cc}
0 & 1 \otimes x^{2} \\
0 & y x \otimes 1+1 \otimes x y
\end{array}\right), \\
& \mathrm{T}^{2}\left(v_{3}\right)=\left(\begin{array}{ccc}
0 & 1 \otimes x^{2} & (y x)^{k-1} \otimes x(y x)^{k-1} \\
0 & y \otimes y & x \otimes x^{2}+x^{2} \otimes x
\end{array}\right) ; \\
& \mathrm{T}^{0}\left(w_{1}\right)=(0,1 \otimes 1), \quad \mathrm{T}^{1}\left(w_{1}\right)=\left(\begin{array}{ccc}
0 & 1 \otimes 1 & \sum_{i=0}^{k-2} x(y x)^{i} \otimes x(y x)^{k-2-i} \\
0 & 0 & \star
\end{array}\right)
\end{aligned}
$$

with

$$
\begin{aligned}
\left(\mathrm{T}^{1}\left(w_{1}\right)\right)_{23} & =\sum_{i=0}^{k-1}(x y)^{i} \otimes(y x)^{k-1-i}+\sum_{i=0}^{k-2}(x y)^{i} \otimes(x y)^{k-1-i}+\sum_{i=1}^{k-1}(y x)^{i} \otimes(y x)^{k-1-i} \\
\mathrm{~T}^{2}\left(w_{1}\right) & =\left(\begin{array}{cccc}
0 & 1 \otimes 1 & 0 & \star \\
0 & 0 & \varphi_{2} & \star
\end{array}\right)
\end{aligned}
$$


with

$$
\begin{aligned}
\left(\mathrm{T}^{2}\left(w_{1}\right)\right)_{14} & =(y x)^{k-1} \otimes x(y x)^{k-2}+x(y x)^{k-2} \otimes(x y)^{k-1}+x(y x)^{k-1} \otimes(x y)^{k-2} \\
\left(\mathrm{~T}^{2}\left(w_{1}\right)\right)_{24} & =\sum_{j=0}^{k-1}(x y)^{j} \otimes(y x)^{k-1-j}+x \otimes y(x y)^{k-2}+(y x)^{k-1} \otimes 1 \\
\mathrm{~T}^{3}\left(w_{1}\right) & =\left(\begin{array}{cccc}
0 & 1 \otimes 1 & 0 & \star \\
0 & 0 & 1 \otimes 1 & \sum_{i=0}^{k-2} x(y x)^{i} \otimes(x y)^{k-2-i}
\end{array}\right)
\end{aligned}
$$

with

$$
\begin{aligned}
\left(\mathrm{T}^{3}\left(w_{1}\right)\right)_{14} & = \begin{cases}0 & \text { if } k>2, \\
y x \otimes x y+(x y)^{2} \otimes 1 & \text { if } k=2 ;\end{cases} \\
\mathrm{T}^{0}\left(w_{2}\right) & =(0, y \otimes 1), \quad \mathrm{T}^{1}\left(w_{2}\right)=\left(\begin{array}{ccc}
0 & 1 \otimes 1 & \star \\
0 & 0 & \star
\end{array}\right)
\end{aligned}
$$

with

$$
\begin{aligned}
\left(\mathrm{T}^{1}\left(w_{2}\right)\right)_{13} & =\sum_{i=1}^{k-1} i x(y x)^{i} \otimes(x y)^{k-1-i}+\sum_{i=1}^{k-1} i(y x)^{i} \otimes x(y x)^{k-1-i}, \\
\left(\mathrm{~T}^{1}\left(w_{2}\right)\right)_{23} & =\sum_{i=0}^{k-2}(i+1) y(x y)^{i} \otimes(y x)^{k-1-i}+\sum_{i=1}^{k-1} i(x y)^{i} \otimes y(x y)^{k-1-i} \\
\mathrm{~T}^{2}\left(w_{2}\right) & =\left(\begin{array}{cccc}
0 & y \otimes 1 & x(y x)^{k-1} \otimes 1 & (y x)^{k-1} \otimes(x y)^{k-1} \\
0 & 0 & y \otimes x+y x \otimes 1 & 0
\end{array}\right), \\
\mathrm{T}^{3}\left(w_{2}\right) & =\left(\begin{array}{cccc}
0 & y \otimes 1 & 1 \otimes x(y x)^{k-1} & 0 \\
0 & 0 & 1 \otimes y & \sum_{i=1}^{k-1} i(y x)^{i} \otimes(x y)^{k-1-i}
\end{array}\right) .
\end{aligned}
$$

The proof of the lemma is a direct verification of the relations $\mu \mathrm{T}^{0}(b)=b, d_{i-1} \mathrm{~T}^{i}(b)$ $=\mathrm{T}^{i-1}(b) d_{i+\operatorname{deg} b}(i>0)$, where $b \in \mathcal{Y}_{1} \backslash\{t\}$ with $\operatorname{deg} b>0$.

Remark 4.4. For what follows, it should be noted that the formulas for the translates of the elements $u_{1}, u_{2}, v_{1}, v_{2}, w_{1}$, and $t$ remain valid for odd values of $k$.

Now the proof of Proposition 4.1 is completed using direct calculations with the matrices described in Lemma 4.3, and we leave this to the reader.

Proposition 4.5. Assume that $k$ is even. The set $\mathcal{Y}_{1}($ see (4.5) $)$ generates $\operatorname{HH}^{*}(R)$ as a K-algebra.

Proof. Let $\mathcal{H}$ denote the $K$-subalgebra of $\operatorname{HH}^{*}(R)$ generated by the set $\mathcal{Y}_{1} \cup\{1\}$ (here 1 denotes the unit element of the algebra $\left.\mathrm{HH}^{*}(R)\right)$. We introduce also the intermediate subalgebra $\mathcal{H}^{\prime}$ generated by the set $\bigcup_{i=0}^{5} \operatorname{HH}^{i}(R)$. First, we show that $\operatorname{HH}^{*}(R)=\mathcal{H}^{\prime}$, and after that we check that $\mathcal{H}^{\prime}=\mathcal{H}$.

The following auxiliary statement is proved by a direct calculation (cf. the proof of Lemma 4.31). 
Lemma 4.6. Put $v:=(1,0) \in \mathrm{HH}^{2}(R)$. The translates $\mathrm{T}^{i}(v), i \leq 5$, can be presented by the maps

$$
\begin{aligned}
\mathrm{T}^{0}(v) & =\left(\begin{array}{lll}
1 \otimes 1 & 0
\end{array}\right) \\
\mathrm{T}^{1}(v) & =\left(\begin{array}{ccc}
1 \otimes 1 & \sum_{i=0}^{k-2} x(y x)^{i} \otimes x(y x)^{k-2-i} \\
0 & \sum_{i=0}^{k-1}(x y)^{i} \otimes(y x)^{k-1-i}
\end{array}\right), \\
\mathrm{T}^{2}(v) & =\left(\begin{array}{ccc}
1 \otimes 1 & 0 & 0 \\
0 & x \otimes 1+1 \otimes x & (x y)^{k-1} \otimes(y x)^{k-1}
\end{array}\right), \\
\mathrm{T}^{3}(v) & =\left(\begin{array}{cccc}
1 \otimes 1 & 0 & 0 & 0 \\
0 & 1 \otimes 1 & \sum_{i=0}^{k-2} x(y x)^{i} \otimes x(y x)^{k-2-i} & 0
\end{array}\right), \\
\mathrm{T}^{4}(v) & =\left(\begin{array}{cccc}
1 \otimes 1 & 0 & 0 & 0 \\
0 & 1 \otimes 1 & 0 & 0 \\
0 & 0 & 1 \otimes 1 & 0
\end{array}\right), \\
\mathrm{T}^{5}(v) & =\left(\begin{array}{cccc}
1 \otimes 1 & 0 & 0 & 0 \\
0 & 1 \otimes 1 & 0 & 0 \\
0 & 0 & 1 \otimes 1 & \sum_{i=0}^{k-2} x(y x)^{i} \otimes x(y x)^{k-2-i} \\
0 & 0 & 0 & \sum_{i=0}^{k-1}(x y)^{i} \otimes(y x)^{k-1-i}
\end{array}\right) .
\end{aligned}
$$

Lemma 4.7. Put $z:=u_{1}^{4} \in \mathrm{HH}^{4}(R)$. The translates $\mathrm{T}^{i}(z), i \leq 3$, can be presented by the maps

$$
\begin{aligned}
\mathrm{T}^{0}(z) & =\left(\begin{array}{lll}
1 \otimes 1 & 0 & 0
\end{array}\right), \\
\mathrm{T}^{1}(z) & =\left(\begin{array}{cccc}
1 \otimes 1 & \sum_{i=0}^{k-2} x(y x)^{i} \otimes x(y x)^{k-2-i} & 0 & 0 \\
0 & \sum_{i=0}^{k-1}(x y)^{i} \otimes(y x)^{k-1-i} & 0 & 0
\end{array}\right), \\
\mathrm{T}^{2}(z) & =\left(\begin{array}{cccc}
1 \otimes 1 & 0 & 0 & 0 \\
0 & x \otimes 1+1 \otimes x & (x y)^{k-1} \otimes(y x)^{k-1} & 0
\end{array}\right), \\
\mathrm{T}^{3}(z) & =\left(\begin{array}{cccc}
1 \otimes 1 & 0 & 0 & 0 \\
0 & 1 \otimes 1 & \sum_{i=0}^{k-2} x(y x)^{i} \otimes x(y x)^{k-2-i} & 0
\end{array}\right) .
\end{aligned}
$$

Moreover, for $i \geq 4$, we have

$$
\mathrm{T}^{i}(z)=\left(\begin{array}{c|c}
\mathrm{id}_{\Lambda^{2}} & \mathrm{O} \\
\hline \mathrm{O} & \mathrm{T}^{i-4}(z)
\end{array}\right) .
$$

Proof. Using the translates $\mathrm{T}^{j}\left(u_{1}\right)$ of the element $u_{1}$ described in Lemma 4.3. we easily show that $z$ can be presented by the cocycle $(1,0,0)$. Then the formulas for $\mathrm{T}^{i}(z)$ with $i \leq 4$ are verified directly. Finally, formula (4.26) is established by induction.

Remark 4.8. We observe that the formulas for translates of the elements $v$ and $z$ presented in Lemmas 4.6 and 4.7 remain valid for any odd $k$.

We continue the proof of Proposition 4.5. Let $f=\left(r_{1}, r_{2}, f^{\prime}\right) \in \operatorname{Ker} \delta^{n} \subset \operatorname{Hom}_{\Lambda}\left(Q_{n}, R\right)$, where $n>5$ and $r_{1}, r_{2} \in R, f^{\prime} \in \operatorname{Hom}_{\Lambda}\left(Q_{n-4}, R\right)$ (here we use the identification $\mathcal{X}^{n}=$ $\operatorname{Hom}_{\Lambda}\left(X_{n}, R\right)$ and $\left.R^{2}\right)$. Since $\delta^{n}(f)=\left(Y * r_{1}, Y * r_{2}, \delta^{n-4}\left(f^{\prime}\right)\right)$, we have $f^{\prime} \in \operatorname{Ker} \delta^{n-4}$.

a) Assume additionally that $n=6$. Let $g \in \mathrm{HH}^{4}(R)$ be an element represented by the cocycle $\left(r_{1}, r_{2}, 0\right)$. Using (4.24), we obtain

$$
\mathrm{T}^{0}(g) \cdot \mathrm{T}^{4}(v)=\left(r_{1} \otimes 1, r_{2} \otimes 1,0\right) \mathrm{T}^{4}(v)=\left(r_{1} \otimes 1, r_{2} \otimes 1,0,0\right) .
$$

Consequently, we have $g \cdot v=\left(r_{1}, r_{2}, 0,0\right)$ and $f-g v=\left(0,0, f^{\prime}\right)$. With the help of Lemma 4.2, we show that $f^{\prime} \cdot t=\left(0,0, f^{\prime}\right)$. Therefore, $f=g v+f^{\prime} t \in \mathcal{H}^{\prime}$. 
b) If $n=7$, then we consider the element $g:=\left(r_{1}, r_{1}, 0,0\right) \in \operatorname{HH}^{5}(R)$. Arguing as above (and using (4.25)), we see that $f=g v+f^{\prime} t \in \mathcal{H}^{\prime}$.

c) Finally, assume that $n \geq 8$. We consider the element $g \in \mathrm{HH}^{n-4}(R)$ represented by the cocycle $\left(r_{1}, r_{2}, \mathrm{O}\right)$. Using (4.26), we obtain

$$
\begin{aligned}
\mathrm{T}^{0}(g) \cdot \mathrm{T}^{n-4}(z) & =\left(r_{1} \otimes 1, r_{2} \otimes 1, \mathrm{O}\right)\left(\begin{array}{c|c}
\mathrm{id}_{\Lambda^{2}} & \mathrm{O} \\
\hline \mathrm{O} & \mathrm{T}^{n-8}(z)
\end{array}\right) \\
& =\left(r_{1} \otimes 1, r_{2} \otimes 1, \mathrm{O}\right) .
\end{aligned}
$$

Hence, $g \cdot z=\left(r_{1}, r_{2}, \mathrm{O}\right) \in \mathrm{HH}^{n}(R)$. As in part a) of the proof, we obtain $f=g z+f^{\prime} t$. Applying the inductive hypothesis to $g$ and $f^{\prime}$, we conclude that $f \in \mathcal{H}^{\prime}$.

Now we prove the inclusion $\mathcal{H}^{\prime} \subset \mathcal{H}$. We remark that, as in the proof of Proposition 4.1. when calculating products of elements of the set $\mathcal{Y}_{1}$, we use the translates listed in Lemma 4.3. By Remark 3.3, we have $\operatorname{HH}^{0}(R) \subset \mathcal{H}$.

Next, the basis elements of the $\mathrm{HH}^{1}(R)$ indicated in Proposition 3.6 satisfy the following relations:

$$
\begin{aligned}
\left(y(x y)^{i}, 0\right) & =p_{1}^{i} u_{3}(0 \leq i \leq k-1), \\
\left(x(y x)^{k-1}, 0\right) & = \begin{cases}p_{2} u_{1} & \text { if } k>2, \\
p_{2} u_{1}+p_{4} u_{2} & \text { if } k=2,\end{cases} \\
\left((x y)^{k}, 0\right) & =p_{4} u_{1}, \quad\left(0, y(x y)^{k-1}\right)=p_{3} u_{2}, \quad\left(0,(x y)^{k}\right)=p_{4} u_{2} .
\end{aligned}
$$

Thus, $\mathrm{HH}^{1}(R) \subset \mathcal{H}$.

A direct calculation with the help of Lemma 4.3 shows that

$$
v= \begin{cases}u_{1}^{2} & \text { if } k>2 \\ u_{1}^{2}+v_{3} & \text { if } k=2\end{cases}
$$

Moreover, we obtain the following relations for the basis elements of $\mathrm{HH}^{2}(R)$ indicated in Proposition 3.10 .

$$
\begin{aligned}
(y x y, 0) & =p_{1} v_{1}, \quad\left(x(y x)^{k-1}, 0\right)=p_{2} u_{1}^{2}, \quad\left((x y)^{k}, 0\right)=p_{4} u_{1}^{2}, \\
(0,1) & =u_{2}^{2}, \quad\left(0, y(x y)^{i}\right)=p_{1}^{i} v_{2} \quad(0 \leq i \leq k-1), \\
\left(0,(x y)^{k-1}\right) & =u_{1} u_{3}+v_{1} .
\end{aligned}
$$

This implies that $\mathrm{HH}^{2}(R) \subset \mathcal{H}$.

As above, we obtain the following relations for the basis elements of $\mathrm{HH}^{3}(R)$ indicated in Proposition 3.14

$$
\begin{aligned}
& (1,0)=u_{1}^{3},(y, 0)=u_{1} v_{1},\left(x(y x)^{k-1}, 0\right)=p_{2} u_{1}^{3},\left((x y)^{k}, 0\right)=p_{4} u_{1}^{3}, \\
& \left(0, y(x y)^{i}, 0\right)=p_{1}^{i} w_{2} \quad(0 \leq i \leq k-1), \\
& (0, x y+y x)=u_{1} v_{2},\left(0, x(y x)^{k-1}\right)=p_{2} w_{1}, \quad\left(0,(x y)^{k}\right)=p_{4} w_{1},
\end{aligned}
$$

and this shows that $\mathrm{HH}^{3}(R) \subset \mathcal{H}$. 
Next, the inclusion $\mathrm{HH}^{4}(R) \subset \mathcal{H}$ is deduced from the following relations for basis elements of $\mathrm{HH}^{4}(R)$ indicated in Proposition 3.19

$$
\begin{aligned}
& (1,0,0)=u_{1}^{4},(y, 0,0)=u_{1}^{2} v_{1}, \\
& \left(x(y x)^{k-1}, 0,0\right)=p_{2} u_{1}^{4},\left((x y)^{k}, 0,0\right)=p_{4} u_{1}^{4}, \\
& (0,1,0)= \begin{cases}u_{1} w_{1} & \text { if } k>2, \\
u_{1} w_{1}+p_{3} t & \text { if } k=2,\end{cases} \\
& \left(0, x(y x)^{k-1}, 0\right)=p_{2} u_{1} w_{1},\left(0,(x y)^{k}, 0\right)=p_{4} u_{1} w_{1}, \\
& (0, y, 0)=u_{3} w_{1}+p_{2} t,
\end{aligned}
$$

and also from the fact that the remaining elements of the mentioned basis have the form $q \cdot t$, where $q$ runs through the basis of the vector space $\mathrm{HH}^{0}(R)$ indicated in (3.2).

Finally, the inclusion $\operatorname{HH}^{5}(R) \subset \mathcal{H}$ is deduced from the following relations for the basis elements of $\mathrm{HH}^{5}(R)$ indicated in Proposition 3.20

$$
\begin{aligned}
& \left(1, \mathrm{O}_{3}\right)=u_{1}^{5},\left(x(y x)^{k-1}, \mathrm{O}_{3}\right)=p_{2} u_{1}^{5},\left((x y)^{k}, \mathrm{O}_{3}\right)=p_{4} u_{1}^{5}, \\
& \left(y, \mathrm{O}_{3}\right)=u_{1}^{3} v_{1},\left(0,1, \mathrm{O}_{2}\right)=u_{1}^{2} w_{1},\left(0, x(y x)^{k-1}, \mathrm{O}_{2}\right)=p_{2} u_{1}^{2} w_{1}, \\
& \left(0,(x y)^{k}, \mathrm{O}_{2}\right)=p_{4} u_{1}^{2} w_{1},
\end{aligned}
$$

and also from the fact that the remaining elements of the mentioned basis have the form $q \cdot t$, where $q$ runs through the basis of the vector space $\operatorname{HH}^{1}(R)$ indicated in Proposition 3.6. This completes the proof of the inclusion $\mathcal{H}^{\prime} \subset \mathcal{H}$.

Let $\mathcal{A}_{1}=K\left[\mathcal{X}_{1}\right] / I_{1}$ be the graded $K$-algebra defined in $\S 1$, where $\mathcal{X}_{1}$ is as in (1.1) and $I_{1}$ is the corresponding ideal of relations (see (1.3)-(1.20) $)$. The (nonzero) images of the monomials in $K\left[\mathcal{X}_{1}\right]$ under the canonical epimorphism $K\left[\mathcal{X}_{1}\right] \rightarrow \mathcal{A}_{1}$ are also called monomials. Any element $a \in \mathcal{A}_{1}$ is represented as a linear combination of monomials (with coefficients in $K$ ). Propositions 4.1 and 4.5 imply that there exists a surjective homomorphism $\varphi: \mathcal{A}_{1} \rightarrow \mathrm{HH}^{*}(R)$ of graded $K$-algebras that takes the generators in the set $\mathcal{X}_{1}$ to the corresponding generators in $\mathcal{Y}_{1}$ (see (4.5)). Note that no ambiguity is caused by employing the same letter to denote elements of the two sets that correspond to each other. Let $\mathcal{A}_{1}=\bigoplus_{m>0} \mathcal{A}_{1}^{m}$ be the direct decomposition of the algebra $\mathcal{A}_{1}$ into homogeneous direct summands. Now, part 1) of Theorem 1.1 is a consequence of the following statement.

Proposition 4.9. For any $m \geq 0$,

$$
\operatorname{dim}_{K} \mathcal{A}_{1}^{m}=\operatorname{dim}_{K} \mathrm{HH}^{m}(R) .
$$

Before proving this proposition, we state the following auxiliary fact.

Lemma 4.10. In the algebra $\mathcal{A}_{1}$, the following relations are true:

$$
\begin{gathered}
p_{1}^{2} u_{1}=p_{1} u_{1}^{2}=p_{3} u_{1}^{2}=p_{1} u_{1} u_{3}=0, \\
p_{1} u_{1} v_{2}=u_{1}^{2} v_{2}=u_{1} u_{3} v_{2}=p_{1} u_{3} w_{1}=u_{2}^{2} v_{1}=u_{3} v_{2} w_{1}=0 .
\end{gathered}
$$

The proof of the lemma is a direct verification of the relations mentioned above, with the use of the defining relations of the algebra $\mathcal{A}_{1}$, and we leave this to the reader. 
Proof of Proposition 4.9, On the polynomial ring $K\left[\mathcal{X}_{1}\right]$, we introduce a lexicographic order such that

$$
w_{2}>u_{3}>w_{1}>v_{3}>v_{2}>v_{1}>u_{2}>u_{1}>t>p_{4}>p_{3}>p_{2}>p_{1} .
$$

By definition, the reduction of an element $f$ in $\mathcal{A}_{1}$ is the process of replacement of some submonomials in $f$ by other elements of $\mathcal{A}_{1}$ in accordance with the following rules ( $a \mapsto b$ means the replacement of every occurrence of the monomial $a$ by the element $b$ ):

$$
\begin{aligned}
& w_{1}^{2} \mapsto u_{1}^{2} t, \quad \quad w_{1} w_{2} \mapsto u_{1} u_{3} t, \\
& v_{2} w_{2} \mapsto p_{1} u_{3} t, \quad v_{2} w_{1} \mapsto p_{3} u_{2} t, \\
& v_{1} w_{2} \mapsto p_{4} u_{1} t, \quad v_{3} w_{1} \mapsto p_{3} u_{1} t, \\
& v_{2}^{2} \mapsto p_{1}^{2} t, \quad u_{1} w_{2} \mapsto u_{3} w_{1}, \\
& p_{1} w_{2} \mapsto u_{3} v_{2}, \quad p_{2} w_{2} \mapsto u_{3} v_{1} \mapsto p_{4} w_{1}, \\
& p_{1} w_{1} \mapsto u_{2} v_{3} \mapsto u_{1} v_{2}, \quad \quad u_{1}^{2} u_{3} \mapsto u_{1} v_{1}+p_{2} w_{1}, \\
& p_{1}^{k-2} u_{3} v_{2} \mapsto p_{3} w_{1} \mapsto u_{1} v_{3} \mapsto u_{2} v_{1}, \quad p_{2} v_{1} \mapsto p_{4} u_{1}^{2}, \\
& p_{3} v_{3} \mapsto p_{2} v_{2} \mapsto p_{1} v_{1} \mapsto p_{4} u_{2}^{2}, \quad p_{2} u_{3} \mapsto p_{4} u_{1}, \\
& p_{1}^{k-1} u_{3} \mapsto p_{2} u_{2} \mapsto p_{3} u_{1}, \quad p_{3} u_{2} \mapsto p_{1} u_{1}, \\
& u_{1} u_{3} w_{1} \mapsto \begin{cases}v_{1} w_{1}+p_{2} u_{1} t & \text { if } k>2, \\
v_{1} w_{1}+\left(p_{2} u_{1}+p_{4} u_{2}\right) t & \text { if } k=2 .\end{cases}
\end{aligned}
$$

Any replacement on the above list is called an elementary step of reduction. Under such a step, any nonzero monomial turns into a strictly smaller element with respect to the lexicographic order. Hence, after finitely many steps, we obtain monomials to which we cannot apply any elementary step of reduction. We say that a presentation of an element $a \in \mathcal{A}_{1}$ as a linear combination of monomials is in normal form if reduction cannot be applied to any of these monomials. Since these elementary steps correspond to some relations satisfied in the algebra $\mathcal{A}_{1}$ (see (1.3)-(1.20) and Lemma 4.10), any element $a \in \mathcal{A}_{1}$ admits at least one presentation in the normal form.

Put $q_{i}=\operatorname{dim}_{K} \mathcal{A}_{1}^{i}$. We denote the number of monomials in $\mathcal{A}_{1}^{i}$ presented in the normal form by $\widetilde{q}_{i}$. It is clear that $\widetilde{q}_{i} \geq q_{i}$. Since there is an epimorphism $\mathcal{A}_{1}^{i} \rightarrow \mathrm{HH}^{i}(R)$, we see that $q_{i} \geq \operatorname{dim}_{K} \mathrm{HH}^{i}(R)$. Consequently, it suffices to show that

$$
\widetilde{q}_{i}=\operatorname{dim}_{K} \mathrm{HH}^{i}(R) .
$$

We shall prove that all (nonzero) monomials having normal form are on the following list.

Monomials of degree $4 n$ :

$$
\begin{aligned}
& \left\{w_{1} u_{1}^{4(n-j)-3} t^{j}\right\}_{j=0}^{n-1},\left\{p_{2} w_{1} u_{1}^{4(n-j)-3} t^{j}\right\}_{j=0}^{n-1},\left\{p_{4} w_{1} u_{1}^{4(n-j)-3} t^{j}\right\}_{j=0}^{n-1}, \\
& \left\{w_{1} v_{1} u_{1}^{4(n-j-1)-1} t^{j}\right\}_{j=0}^{n-2},\left\{v_{1} u_{1}^{4(n-j)-2} t^{j}\right\}_{j=0}^{n-1},\left\{u_{1}^{4(n-j)} t^{j}\right\}_{j=0}^{n} \\
& \left\{p_{2} u_{1}^{4(n-j)} t^{j}\right\}_{j=0}^{n},\left\{p_{4} u_{1}^{4(n-j)} t^{j}\right\}_{j=0}^{n},\left\{p_{1}^{i} t^{n}\right\}_{i=1}^{k-1}, p_{3} t^{n}, \\
& u_{3} w_{1} t^{n-1} ;
\end{aligned}
$$


monomials of degree $4 n+1$ :

$$
\begin{aligned}
& \left\{w_{1} u_{1}^{4(n-j)-2} t^{j}\right\}_{j=0}^{n-1},\left\{p_{2} w_{1} u_{1}^{4(n-j)-2} t^{j}\right\}_{j=0}^{n-1},\left\{p_{4} w_{1} u_{1}^{4(n-j)-2} t^{j}\right\}_{j=0}^{n-1}, \\
& \left\{w_{1} v_{1} u_{1}^{4(n-j-1)} t^{j}\right\}_{j=0}^{n-1},\left\{v_{1} u_{1}^{4(n-j)-1} t^{j}\right\}_{j=0}^{n-1},\left\{u_{1}^{4(n-j)+1} t^{j}\right\}_{j=0}^{n}, \\
& \left\{p_{2} u_{1}^{4(n-j)+1} t^{j}\right\}_{j=0}^{n},\left\{p_{4} u_{1}^{4(n-j)+1} t^{j}\right\}_{j=0}^{n}, u_{2} t^{n}, p_{4} u_{2} t^{n}, p_{1} u_{1} t^{n}, p_{3} u_{1} t^{n}, \\
& \left\{u_{3} t^{n} p_{1}^{i}\right\}_{i=0}^{k-2}
\end{aligned}
$$

monomials of degree $4 n+2$ :

$$
\begin{aligned}
& \left\{w_{1} v_{1} u_{1}^{4(n-j)-3} t^{j}\right\}_{j=0}^{n-1},\left\{w_{1} u_{1}^{4(n-j)-1} t^{j}\right\}_{j=0}^{n-1},\left\{p_{2} w_{1} u_{1}^{4(n-j)-1} t^{j}\right\}_{j=0}^{n-1}, \\
& \left\{p_{4} w_{1} u_{1}^{4(n-j)-1} t^{j}\right\}_{j=0}^{n-1},\left\{v_{1} u_{1}^{4(n-j)} t^{j}\right\}_{j=0}^{n},\left\{u_{1}^{4(n-j)+2} t^{j}\right\}_{j=0}^{n}, \\
& \left\{p_{2} u_{1}^{4(n-j)+2} t^{j}\right\}_{j=0}^{n},\left\{p_{4} u_{1}^{4(n-j)+2} t^{j}\right\}_{j=0}^{n}, p_{1} u_{1} t^{n}, u_{2}^{2} t^{n}, p_{4} u_{2}^{2} t^{n}, \\
& \left\{v_{2} t^{n} p_{1}^{i}\right\}_{i=0}^{k-2}, u_{3} u_{1} t^{n}, v_{3} t^{n}
\end{aligned}
$$

monomials of degree $4 n+3$ :

$$
\begin{aligned}
& \left\{w_{1} v_{1} u_{1}^{4(n-j)-3} t^{j}\right\}_{j=0}^{n-1},\left\{w_{1} u_{1}^{4(n-j)} t^{j}\right\}_{j=0}^{n},\left\{p_{2} w_{1} u_{1}^{4(n-j)} t^{j}\right\}_{j=0}^{n}, \\
& \left\{p_{4} w_{1} u_{1}^{4(n-j)} t^{j}\right\}_{j=0}^{n},\left\{v_{1} u_{1}^{4(n-j)+1} t^{j}\right\}_{j=0}^{n},\left\{u_{1}^{4(n-j)+3} t^{j}\right\}_{j=0}^{n} \\
& \left\{p_{2} u_{1}^{4(n-j)+3} t^{j}\right\}_{j=0}^{n},\left\{p_{4} u_{1}^{4(n-j)+3} t^{j}\right\}_{j=0}^{n}, w_{2} t^{n}, v_{1} u_{2} t^{n}, v_{2} u_{1} t^{n}, \\
& \left\{u_{3} v_{2} t^{n} p_{1}^{i}\right\}_{i=0}^{k-3}
\end{aligned}
$$

(here, $n \in \mathbb{N} \cup\{0\}$ ). It is easily seen that all monomials on this list have normal form.

Let

$$
f=\left(w_{2}\right)^{\alpha_{2}} u_{3}^{\beta_{3}} w_{1}^{\alpha_{1}} v_{3}^{\gamma_{3}} v_{2}^{\gamma_{2}} v_{1}^{\gamma_{1}} u_{2}^{\beta_{2}} u_{1}^{i} t^{j} p_{4}^{\varepsilon_{4}} p_{3}^{\varepsilon_{3}} p_{2}^{\varepsilon_{2}} p_{1}^{\ell}
$$

be a nonzero monomial represented in the normal form. Using relations satisfied in the algebra $\mathcal{A}_{1}$, we see that in (4.49) we have

$$
\begin{gathered}
\alpha_{1}, \alpha_{2}, \beta_{3}, \gamma_{1}, \gamma_{2}, \gamma_{3}, \varepsilon_{2}, \varepsilon_{3}, \varepsilon_{4} \in\{0,1\}, \beta_{2} \in\{0,1,2\}, \\
i, j, \ell \in \mathbb{N} \cup\{0\}, \text { and } \ell \leq k-1 .
\end{gathered}
$$

Next, we consider several cases successively.

1) Assume that the monomial $f$ involves the factor $w_{2}$. Then, by some steps of reduction and monomial relations satisfied in the algebra $\mathcal{A}_{1}$ (see (1.3)-(1.20) and Lemma 4.10), we conclude that $f$ does not contain $u_{3}, w_{1}, v_{3}, v_{2}, v_{1}, u_{2}, u_{1}, p_{4}, p_{3}, p_{2}, p_{1}$. Consequently,

$$
f=w_{2} t^{j} .
$$

2) Assume that $f$ does not involve $w_{2}$, but involves $u_{3}$. As above, we conclude that $f$ does not contain $v_{3}, v_{1}, u_{2}, p_{2}, p_{3}, p_{4}$. Hence,

$$
f=u_{3} w_{1}^{\alpha_{1}} v_{2}^{\gamma_{2}} v_{1}^{\gamma_{1}} u_{1}^{i} t^{j} p_{1}^{\ell} .
$$

Here $i \leq 1$, because of the reduction $u_{1}^{2} u_{3} \mapsto u_{1} v_{1}+p_{2} w_{1}$; furthermore, $\ell \leq k-2$ (the reduction $p_{1}^{k-1} u_{3} \mapsto p_{3} u_{1}$ ), and for $\gamma_{2}=1$ we have $\ell \leq k-3$ (the reduction $\left.p_{1}^{k-2} u_{3} v_{2} \mapsto u_{2} v_{1}\right)$. Also, $\alpha_{1}+\gamma_{2}+i \leq 1$ (because $u_{1} u_{3} v_{2}=u_{3} v_{2} w_{1}=0$ and the 
monomial $u_{1} u_{3} w_{1}$ can be reduced). Finally, if $\alpha_{1}=1$, then $\ell=0$ (because $p_{1} u_{3} w_{1}=0$ ). Consequently, $f$ coincides with one of the following monomials:

$$
u_{3} t^{j} p_{1}^{\ell}(0 \leq \ell \leq k-2), u_{3} v_{2} t^{j} p_{1}^{\ell}(0 \leq \ell \leq k-3), u_{3} w_{1} t^{j}, u_{3} u_{1} t^{j} .
$$

3) Assume that $f$ involves neither $w_{2}$ nor $u_{3}$, but involves $w_{1}$. Arguing as above, we conclude that $f$ does not contain $v_{3}, v_{2}, u_{2}, p_{3}, p_{1}$. Moreover, in (4.49) we have $\gamma_{1}+\varepsilon_{4}+$ $\varepsilon_{2} \leq 1$ (because $p_{4} v_{1}=p_{2} p_{4}=0$ and we have the reduction $p_{2} v_{1} \mapsto p_{4} u_{1}^{2}$ ). Consequently, $f$ coincides with one of the following monomials:

$$
w_{1} u_{1}^{i} t^{j}, p_{2} w_{1} u_{1}^{i} t^{j}, p_{4} w_{1} u_{1}^{i} t^{j}, w_{1} v_{1} u_{1}^{i} t^{j}
$$

with $i, j \in \mathbb{N} \cup\{0\}$.

4) Assume that $w_{2}, u_{3}, w_{1}$ do not occur and $v_{3}$ occurs in $f$. Then it is easily seen that $f$ does not involve $v_{2}, v_{1}, u_{2}, u_{1}, p_{4}, p_{3}, p_{2}, p_{1}$. Consequently, $f$ is a monomial of the form $f=v_{3} t^{j}$.

5) Assume that $w_{2}, u_{3}, w_{1}, v_{3}$ do not occur and $v_{2}$ occurs in $f$. Then $f$ does not involve $v_{1}, u_{2}, p_{4}, p_{3}, p_{2}$ either. Hence, $f=v_{2} u_{1}^{i} t^{j} p_{1}^{\ell}$, where $\ell \leq k-2$ (because $p_{1}^{k-1} v_{2}=0$ ) and $i \leq 1$ (because $u_{1}^{2} v_{2}=0$ ); moreover, if $i=1$, then $\ell=0$ (because $p_{1} u_{1} v_{2}=0$ ). Consequently, $f$ coincides with one of the following monomials:

$$
v_{2} t^{j} p_{1}^{\ell}(0 \leq \ell \leq k-2), v_{2} u_{1} t^{j} .
$$

6) Assume that $w_{2}, u_{3}, w_{1}, v_{3}, v_{2}$ do not occur and $v_{1}$ occurs in $f$. Then $f$ does not involve $p_{4}, p_{3}, p_{2}, p_{1}$. In (4.49) we have $\beta_{2} \leq 1$ because $u_{2}^{2} v_{1}=0$; moreover, if $i \geq 1$, then $\beta_{2}=0$ (because $u_{1} u_{2}=0$ ). Consequently, $f$ coincides with one of the following monomials:

$$
v_{1} u_{1}^{i} t^{j}, v_{1} u_{2} t^{j}(i, j \in \mathbb{N} \cup\{0\}) .
$$

7) Assume that $w_{2}, u_{3}, w_{1}, v_{3}, v_{2}, v_{1}$ do not occur and $u_{2}$ occurs in $f$. Then $f$ does not involve $u_{1}, p_{3}, p_{2}, p_{1}$. Consequently, $f$ is one of the following monomials:

$$
u_{2} t^{j}, p_{4} u_{2} t^{j}, u_{2}^{2} t^{j}, p_{4} u_{2}^{2} t^{j} .
$$

8) Assume that $w_{2}, u_{3}, w_{1}, v_{3}, v_{2}, v_{1}, u_{2}$ do not occur and $u_{1}$ occurs in $f$. Then in (4.49) we have $\ell \leq 1$ (because $p_{1}^{2} u_{1}=0$ ) and $\ell+\sum_{r=2}^{4} \varepsilon_{r} \leq 1$ (because $p_{r} p_{s}=0$ for $r \neq s$ ); moreover, if $i \geq 2$, then $\varepsilon_{3}=\ell=0$ (because $p_{1} u_{1}^{2}=p_{3} u_{1}^{2}=0$ ). Consequently, $f$ is one of the following monomials:

$$
u_{1}^{i} t^{j}, p_{2} u_{1}^{i} t^{j}, p_{4} u_{1}^{i} t^{j}, p_{1} u_{1} t^{j}, p_{3} u_{1} t^{j}
$$

with $i \in \mathbb{N}, j \in \mathbb{N} \cup\{0\}$.

9) Finally, assume that $w_{2}, u_{3}, w_{1}, v_{3}, v_{2}, v_{1}, u_{2}, u_{1}$ do not occur in $f$. In this case, clearly, $f$ coincides with one of the monomials:

$$
t^{j} p_{1}^{\ell}(0 \leq \ell \leq k-1), t^{j} p_{2}, t^{j} p_{3}, t^{j} p_{4} .
$$

Therefore, we have shown that any monomial having normal form is on the list above. It is easily seen that

$$
\begin{array}{ll}
\widetilde{q}_{4 n}=8 n+k+3, & \widetilde{q}_{4 n+1}=8 n+k+6, \\
\widetilde{q}_{4 n+2}=8 n+k+7, & \widetilde{q}_{4 n+3}=8 n+k+8 .
\end{array}
$$

Now (4.32) follows from Corollary 3.18 
Case 2. Now we assume that $k$ is odd. We consider the following homogeneous elements of $\mathrm{HH}^{*}(R)$ :

$\begin{array}{ll}\text { - of degree } 0: & p_{1}, p_{2}, p_{3}, p_{4} \text { in (3.5); } \\ \text { - of degree 1: } & u_{1}, u_{2} \text { in (4.1) and } u_{3}^{\prime}:=(0, x) \text {; } \\ \text { - of degree 2: } & v_{1}, v_{2} \text { in (4.2); } \\ \text { - of degree 3: } & w_{1}, w_{2} \text { in (4.3); } \\ \text { - of degree } 4: & t \text { in (4.4). }\end{array}$

Proposition 4.11. Assume that $k$ is odd. In the algebra $\mathrm{HH}^{*}(R)$, the elements of the set

$$
\mathcal{Y}_{2}=\left\{p_{1}, p_{2}, p_{3}, p_{4}, u_{1}, u_{2}, u_{3}^{\prime}, v_{1}, v_{2}, w_{1}, w_{2}, t\right\}
$$

satisfy the following relations: (4.6), (4.7), (4.9), (4.10), (4.13), (4.14), (4.17), (4.19), (4.22), and the relations

$$
\begin{aligned}
& \text { (4.51) } p_{2} u_{2}=p_{1}^{k-1} u_{3}^{\prime}, p_{4} u_{2}=p_{3} u_{3}^{\prime}, p_{2} u_{3}^{\prime}=p_{4} u_{3}^{\prime}=0 ; \\
& \text { (4.52) } p_{2} v_{2}=p_{3} u_{2} u_{3}^{\prime}, u_{1} u_{2}=p_{1}^{k-2} v_{2},\left(u_{3}^{\prime}\right)^{2}=0 ; \\
& (4.53) \quad p_{1} w_{1}=u_{2}^{2} u_{3}^{\prime}, p_{3} w_{1}=p_{1}^{k-1} w_{2}, p_{4} w_{1}=u_{3}^{\prime} v_{1}, p_{1} w_{2}=u_{3}^{\prime} v_{2}, p_{2} w_{1}=u_{1}^{2} u_{3}^{\prime} ; \\
& (4.54) \quad u_{2} w_{1}=p_{1}^{k-1} t, u_{2} w_{2}=p_{3} t, u_{3}^{\prime} w_{1}=p_{2} t, u_{3}^{\prime} w_{2}=p_{4} t ; \\
& (4.55) \\
& v_{1} w_{2}=0, v_{2} w_{2}=p_{1} u_{3}^{\prime} t, u_{1}^{2} w_{2}=v_{1} w_{1} ; \\
& \text { (4.56) } w_{1} w_{2}=v_{1} t .
\end{aligned}
$$

As in the proof of Proposition 4.1, we need to compute the translates for the elements in $\mathcal{Y}_{2}$ for which they were not described earlier (see Remark 4.4).

Lemma 4.12. For the role of the translates of the elements $u_{3}^{\prime}$ and $w_{2}$, we can take the homomorphisms determined by the following matrices:

$$
\begin{gathered}
\mathrm{T}^{0}\left(u_{3}^{\prime}\right)=(0, x \otimes 1), \quad \mathrm{T}^{1}\left(u_{3}^{\prime}\right)=\left(\begin{array}{cc}
0 & \sum_{i=1}^{k-2} i(y x)^{i} \otimes(x y)^{k-1-i} \\
0 & x \otimes 1+\sum_{i=1}^{k-2} i y(x y)^{i} \otimes y(x y)^{k-2-i}
\end{array}\right), \\
\mathrm{T}^{0}\left(w_{2}\right)=(0, y \otimes 1), \quad \mathrm{T}^{1}\left(w_{2}\right)=\left(\begin{array}{ccc}
0 & 1 \otimes 1 & \star \\
0 & 0 & \star
\end{array}\right)
\end{gathered}
$$

with

$$
\begin{aligned}
\left(\mathrm{T}^{1}\left(w_{2}\right)\right)_{13} & =\sum_{i=0}^{k-1}(i+1) x(y x)^{i} \otimes(x y)^{k-1-i}+\sum_{i=0}^{k-1}(i+1)(y x)^{i} \otimes x(y x)^{k-1-i}, \\
\left(\mathrm{~T}^{1}\left(w_{1}\right)\right)_{23} & =\sum_{i=0}^{k-1}(i+1)(x y)^{i} \otimes y(x y)^{k-1-i}+\sum_{i=1}^{k-2} i y(x y)^{i} \otimes(y x)^{k-1-i}, \\
\mathrm{~T}^{2}\left(w_{2}\right) & =\left(\begin{array}{cccc}
0 & y \otimes 1 & \rho & 0 \\
0 & 0 & y \otimes x+y x \otimes 1 & 1 \otimes y(x y)^{k-1}
\end{array}\right), \\
\mathrm{T}^{3}\left(w_{2}\right) & =\left(\begin{array}{cccc}
0 & y \otimes 1 & 0 & 0 \\
0 & 0 & 1 \otimes y & \star
\end{array}\right)
\end{aligned}
$$

with

$$
\left(\mathrm{T}^{3}\left(w_{2}\right)\right)_{24}=\sum_{i=0}^{k-1}(i+1)(y x)^{i} \otimes(x y)^{k-1-i} .
$$

The proof of this lemma and the derivation of Proposition 4.11 from it are left to the reader. 
Proposition 4.13. Assume that $k$ is odd. The set $\mathcal{Y}_{2}$ (see (4.50) $)$ generates $\mathrm{HH}^{*}(R)$ as a K-algebra.

Proof. Let $\mathcal{H}$ denote a $K$-subalgebra of $\operatorname{HH}^{*}(R)$ generated by the set $\mathcal{Y}_{2} \cup\{1\}$. As in the proof of Proposition 4.5 we introduce also the subalgebra $\mathcal{H}^{\prime}$ generated by the set $\bigcup_{i=0}^{5} \operatorname{HH}^{i}(R)$. Then the proof of the fact that $\operatorname{HH}^{*}(R)=\mathcal{H}^{\prime}$ repeats word for word the corresponding part of the proof of Proposition 4.5.

Now, we establish the inclusion $\mathcal{H}^{\prime} \subset \mathcal{H}$. The inclusion $\operatorname{HH}^{0}(R) \subset \mathcal{H}$ follows from Remark 3.3. The inclusion $\operatorname{HH}^{1}(R) \subset \mathcal{H}$ is derived from (4.27) and the relations

$$
\left(y(x y)^{i}, 0\right)=p_{1}^{i} u_{3}^{\prime}(0 \leq i \leq k-1), \quad\left(x(y x)^{k-1}, 0\right)=p_{2} u_{1} .
$$

Next, in the group $\mathrm{HH}^{2}(R)$, relations (4.28), (4.29), and

$$
(0, x)=u_{2} u_{3}^{\prime}, \quad\left(0,(x y)^{k-1}\right)=u_{1} u_{3}^{\prime}
$$

are satisfied. This proves the inclusion $\operatorname{HH}^{2}(R) \subset \mathcal{H}$. Finally, the proof of the fact that $\mathrm{HH}^{i}(R) \subset \mathcal{H}$ for $i=3,4,5$ repeats word for word the corresponding part of the proof of Proposition 4.5. except that, in the group $\mathrm{HH}^{4}(R)$, instead of (4.30) we use the relation $(0, y, 0)=u_{1} w_{2}$.

Let $\mathcal{A}_{2}=K\left[\mathcal{X}_{2}\right] / I_{2}$ be the graded $K$-algebra defined in $\S 1$, where $I_{2}$ is the corresponding ideal of relations. Propositions 4.11 and 4.13 imply that there exists a surjective homomorphism $\varphi: \mathcal{A}_{2} \rightarrow \mathrm{HH}^{*}(R)$ of graded $K$-algebras that takes the generators in the set $\mathcal{X}_{2}$ to the corresponding generators in $\mathcal{Y}_{2}$. Let $\mathcal{A}_{2}=\bigoplus_{m>0} \mathcal{A}_{2}^{m}$ be the direct decomposition of the algebra $\mathcal{A}_{2}$ into homogeneous direct summands. Now, part 2) of Theorem 1.1 is a consequence of the following statement.

Proposition 4.14. For any $m \geq 0$ we have

$$
\operatorname{dim}_{K} \mathcal{A}_{2}^{m}=\operatorname{dim}_{K} \operatorname{HH}^{m}(R) .
$$

Proof. The proof of (4.57) is similar to that of Proposition 4.9. First we need the following auxiliary fact, which is established by direct calculations.

Lemma 4.15. In the algebra $\mathcal{A}_{2}$, the following relations are satisfied:

$$
\begin{aligned}
p_{1}^{2} u_{1}= & p_{1} u_{1}^{2}=p_{3} u_{1}^{2}=p_{4} u_{1} u_{2}=p_{1} u_{1} v_{2}=0, \\
u_{1}^{2} u_{2}= & u_{1} u_{2}^{2}=u_{1} u_{2} v_{1}=u_{1} u_{3}^{\prime} v_{2}=u_{2}^{2} v_{1}=0, \\
& p_{1} v_{1}=p_{4} u_{2}^{2}, u_{1} u_{2} u_{3}^{\prime}=u_{2} v_{1} .
\end{aligned}
$$

Next, on the polynomial ring $K\left[\mathcal{X}_{2}\right]$ we introduce a lexicographic order such that

$$
w_{2}>u_{3}^{\prime}>w_{1}>v_{2}>v_{1}>u_{2}>u_{1}>t>p_{4}>p_{3}>p_{2}>p_{1} .
$$

Then we consider the following list of elementary steps of reduction:

$$
\begin{array}{ll}
w_{1}^{2} \mapsto u_{1}^{2} t, & w_{1} w_{2} \mapsto v_{1} t, \\
u_{1}^{2} w_{2} \mapsto v_{1} w_{1}, & v_{2} w_{2} \mapsto p_{1} u_{3}^{\prime} t, \\
v_{2} w_{1} \mapsto p_{3} u_{2} t, & u_{3}^{\prime} w_{2} \mapsto p_{4} t, \\
u_{3}^{\prime} w_{1} \mapsto p_{2} t, & u_{2} w_{1} \mapsto p_{1}^{k-1} t, \\
u_{2} w_{2} \mapsto p_{3} t, & v_{2}^{2} \mapsto p_{1}^{2} t, \\
p_{1} w_{2} \mapsto u_{3}^{\prime} v_{2}, & p_{2} w_{2} \mapsto u_{3}^{\prime} v_{1} \mapsto p_{4} w_{1}, \\
u_{2}^{2} u_{3}^{\prime} \mapsto p_{1} w_{1} \mapsto u_{1} v_{2}, & p_{1}^{k-1} w_{2} \mapsto p_{3} w_{1} \mapsto u_{2} v_{1}, \\
u_{1}^{2} u_{3}^{\prime} \mapsto p_{2} w_{1}, & u_{1} u_{2} u_{3}^{\prime} \mapsto u_{2} v_{1}, \\
p_{3} u_{2} u_{3}^{\prime} \mapsto p_{2} v_{2} \mapsto p_{1} v_{1} \mapsto p_{4} u_{2}^{2}, & p_{2} v_{1} \mapsto p_{4} u_{1}^{2}, \\
p_{1}^{k-2} v_{2} \mapsto u_{1} u_{2}, & p_{3} u_{3}^{\prime} \mapsto p_{2} u_{2}, \\
p_{1}^{k-1} u_{3}^{\prime} \mapsto p_{2} u_{2} \mapsto p_{3} u_{1}, & p_{3} u_{2} \mapsto p_{1} u_{1} .
\end{array}
$$


As in the proof of Proposition 4.9, we define a normal form of an element $f \in \mathcal{A}_{2}$ (with respect to the steps of reduction listed in (4.58) ) and then we check that any element $f \in \mathcal{A}_{2}$ admits at least one normal form. Finally, analyzing successively several cases we prove that all (nonzero) monomials presented in the normal form belong to the following list.

Monomials of degree $4 n$ :

a) the monomials in (4.33)-4.35), b) $w_{2} u_{1} t^{n-1}$;

monomials of degree $4 n+1$ :

a) the monomials in (4.37)-(4.39), b) $\left\{u_{3}^{\prime} t^{n} p_{1}^{i}\right\}_{i=0}^{k-2}$;

monomials of degree $4 n+2$ :

a) the monomials in (4.41)-(4.43), b) $\left\{v_{2} t^{n} p_{1}^{i}\right\}_{i=0}^{k-3}, u_{3}^{\prime} u_{1} t^{n}, u_{3}^{\prime} u_{2} t^{n}, u_{2} u_{1} t^{n}$; monomials of degree $4 n+3$ :

a) the monomials in (4.45)-(4.47), b) $\left\{u_{3}^{\prime} v_{2} t^{n} p_{1}^{i}\right\}_{i=0}^{k-3}$

(here, $n \in \mathbb{N} \cup\{0\}$ ). Now, as in the proof of Proposition 4.9, we can obtain (4.57). The details of the corresponding arguments are left to the reader.

\section{REFERENCES}

[1] S. F. Siegel and S. J. Witherspoon, The Hochschild cohomology ring of a group algebra, Proc. London Math. Soc. (3) 79 (1999), 131-157. MR.1687539 (2000b:16016)

[2] K. Erdmann and Th. Holm, Twisted bimodules and Hochschild cohomology for self-injective algebras of class $A_{n}$, Forum Math. 11 (1999), 177-201. MR1680594 (2001c:16018)

[3] A. I. Generalov, Hochschild cohomology of algebras of dihedral type, I: The family $D(3 \mathcal{K})$ in characteristic 2, Algebra i Analiz 16 (2004), no. 6, 53-122; English transl., St. Petersburg Math. J. 16 (2005), no. 6, 961-1012. MR2117449 (2005i:16013)

[4] K. Erdmann, Blocks of tame representation type and related algebras, Lecture Notes in Math., vol. 1428, Springer-Verlag, Berlin, 1990. MR 1064107 (91c:20016)

[5] A. I. Generalov, Hochschild cohomology of algebras of quaternion type, I: Generalized quaternion groups, Algebra i Analiz 18 (2006), no. 1, 55-107; English transl., St. Petersburg Math. J. 18 (2007), no. 1, 37-76. MR2225213 (2007d:16021)

[6] A. I. Generalov, A. A. Ivanov, and S. O. Ivanov, Hochschild cohomology of algebras of quaternion type. II. The family $Q(2 \mathcal{B})_{1}$ in characteristic 2, Zap. Nauchn. Sem. S.-Peterburg. Otdel. Mat. Inst. Steklov. (POMI) 349 (2007), 53-134; English transl., J. Math. Sci. (New York) 151 (2008), no. 3, 2961-3009.

[7] A. I. Generalov, Hochschild cohomology of algebras of quaternion type. III. Algebras with a small parameter, Zap. Nauchn. Sem. S.-Peterburg. Otdel. Mat. Inst. Steklov. (POMI) 356 (2008), 46-84; English transl. in J. Math. Sci. (New York).

[8] K. Erdmann and A. Skowroński, The stable Calabi-Yau dimension of tame symmetric algebras, J. Math. Soc. Japan 58 (2006), no. 1, 97-128. MR2204567 (2007b:16037)

[9] N. Yu. Kosovskaya, Bimodule resolution for Liu-Schulz algebras, Zap. Nauchn. Sem. S.-Peterburg. Otdel. Mat. Inst. Steklov. (POMI) 321 (2005), 213-223; English transl., J. Math. Sci. (New York) 136 (2006), no. 3, 3951-3956. MR2138419(2006e:16012)

[10] A. I. Generalov and N. Yu. Kosovskaya, Hochschild cohomology of the Liu-Schulz algebras, Algebra i Analiz 18 (2006), no. 4, 39-82; English transl., St. Petersburg. Math. J. 18 (2007), no. 4, 539-572. MR 2262583(2007e:16013)

[11] A. I. Generalov, The Hochschild cohomology of the integer group ring of a dihedral group. I. The even case, Algebra i Analiz 19 (2007), no. 5, 70-123; English transl., St. Petersburg. Math. J. 19 (2008), no. 5, 723-763. MR2381942(2009e:16015)

[12] T. Hayami, Hochschild cohomology ring of the integral group ring of dihedral groups, Tsukuba J. Math. 31 (2007), 99-127. MR.2337122 (2008d:16016)

[13] K. Erdmann, Th. Holm, and N. Snashall, Twisted bimodules and Hochschild cohomology for selfinjective algebras of class $A_{n}$. II, Algebr. Represent. Theory 5 (2002), 457-482. MR1935856 (2004a:16013) 
[14] A. I. Generalov and M. A. Kachalova, Bimodule resolution of the Möbius algebra, Zap. Nauchn. Sem. S.-Peterburg. Otdel. Mat. Inst. Steklov. (POMI) 321 (2005), 36-66; English transl., J. Math. Sci. (New York) 136 (2006), no. 3, 3850-3866. MR2138411 (2006a:16014)

[15] M. A. Kachalova, The Hochschild cohomology for the Möbius algebra, Zap. Nauchn. Sem. S.Peterburg. Otdel. Mat. Inst. Steklov. (POMI) 330 (2006), 173-200; English transl., J. Math. Sci. (New York) 140 (2007), no. 5, 699-715. MR2253573 (2007f:16016)

[16] Yu. V. Volkov and A. I. Generalov, Hochschild cohomology for self-injective algebras of tree class $D_{n}$. I, Zap. Nauchn. Sem. S.-Peterburg. Otdel. Mat. Inst. Steklov. (POMI) 343 (2007), 121-182; English transl., J. Math. Sci. (New York) 147 (2007), no. 5, 7042-7073. MR2469415

[17] Th. Holm, Hochschild cohomology of tame blocks, J. Algebra 271 (2004), 798-826. MR.2025551 (2005c:20019)

[18] S. Eilenberg and S. MacLane, Cohomology theory in abstract groups. I, Ann. of Math. (2) 48 (1947), 51-78. MR0019092 (8:367f)

[19] H. Cartan and S. Eilenberg, Homological algebra, Princeton Univ. Press, Princeton, NJ, 1956. MR $0077480(17: 1040 \mathrm{e})$

[20] M. Gerstenhaber, The cohomology structure of an associative ring, Ann. of Math. (2) 78 (1963), 267-288. MR 0161898 (28:5102)

[21] M. Gerstenhaber and S. D. Schack, Algebraic cohomology and deformation theory, Deformation Theory of Algebras and Structures, and Applications (Il Ciocco, 1986) ( M. Hazewinkel, M. Gerstenhaber, eds.), NATO Adv. Sci. Inst. Ser. C Math. Phys. Sci., vol. 247, Kluwer Acad. Publ., Dordrecht, 1988, pp. 11-264. MR0981619 (90c:16016)

Department of Mathematics and Mechanics, St. Petersburg State University, UniverSitetskil Prospekt 28, Petrodvorets, St. Petersburg 198504, Russia

E-mail address: general@pdmi.ras.ru

Received 1/SEP/2008

Translated by THE AUTHOR 